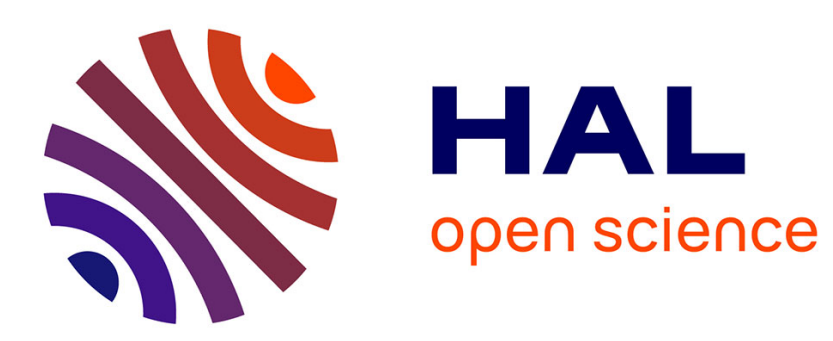

\title{
Les poignards et les coutelas dans les sépultures gallo-romaines du Limousin
}

Guy Lintz, Dominique Vuaillat

\section{To cite this version:}

Guy Lintz, Dominique Vuaillat. Les poignards et les coutelas dans les sépultures gallo-romaines du Limousin. Gallia - Fouilles et monuments archéologiques en France métropolitaine, 1988, 45, pp.165188. 10.3406/galia.1987.2885 . hal-01939603

\section{HAL Id: hal-01939603 https://hal.science/hal-01939603}

Submitted on 14 Jan 2020

HAL is a multi-disciplinary open access archive for the deposit and dissemination of scientific research documents, whether they are published or not. The documents may come from teaching and research institutions in France or abroad, or from public or private research centers.
L'archive ouverte pluridisciplinaire HAL, est destinée au dépôt et à la diffusion de documents scientifiques de niveau recherche, publiés ou non, émanant des établissements d'enseignement et de recherche français ou étrangers, des laboratoires publics ou privés.

\section{(이) $\$$}

Distributed under a Creative Commons Attribution - NonCommercial - NoDerivatives| 4.0 


\title{
LES POIGNARDS ET LES COUTELAS DANS LES SÉPULTURES GALLO-ROMAINES DU LIMOUSIN
}

\author{
par Guy LINTZ et Dominique VUAILLAT
}

De nouvelles découvertes de "glaives" à lame ondulée dans les sépultures gallo-romaines $\mathrm{du} \mathrm{Li-}$ mousin nous ont incités à revoir l'inventaire de ce type d'objets publié en 1941 par le Commandant Martignon.

Ce nouvel inventaire tient compte des découvertes récentes et anciennes, y compris celles qui peuvent être considérées comme douteuses. Certains objets mentionnés en bibliographie ont aujourd'hui disparu des collections publiques ou privées. C'est la raison pour laquelle vingt et un exemplaires seulement ont pu être pris en compte dans l'étude typologique de ces armes. Les données descriptives ont été vérifiées sur les objets eux-mêmes chaque fois que cela a été possible. A défaut, nous avons utilisé les seules données bibliographiques lorsqu'elles étaient suffisamment précises.

Presque tous ces objets proviennent de sépultures en coffres funéraires qui constituent la structure type de la tombe gallo-romaine en Limousin (Lintz, 1981a). Le coffre funéraire, taillé dans la roche locale, possède une forme cylindrique ou prismatique el comprend deux parties : le socle dont la face supérieure est creusée d'un réceptacle entouré d'un bourrelet et le couvercle. Les ossements calcinés, sont recueillis dans une urne déposée dans le réceptacle. L'urne peut toutefois être absente; les ossements se trouvent alors à même la pierre.

Le coffre funéraire, était placé dans une fosse le plus souvent comblée à l'aide du résidu provenant du bûcher de la crémation. Outre les cendres et les charbons de bois, le résidu de la crémation comprend les restes d'objets brisés et brûlés sur le bûcher (tessons de céramique, verre fondu ...). Lorsque, dans la tombe, la position des coutelas est précisée, ils sont dissociés du résidu de la crémation. Les objets miniaturisés se trouvent à l'intérieur du coffre funéraire, dans l'urne, ou à côté. Le coutelas de Saint-Martin-Terressus (Haute-Vienne) était posé sur une banquette taillée dans la paroi de la fosse (fig. 1).

\section{TYPOLOGIE}

Nous rappellerons pour mémoire la définition que le Commandant Martignon a donnée de ces armes (Martignon, 1941, p. 408-409):

1, lame : pointue, tranchante d'un seul côté. Dos légèrement convexe. Taillant, concave dans la partie avoisinant la poignée, convexe dans la partie avoisinant la pointe. Pointe sensiblement dans le prolongement de la poignée. En résumé, forme générale rappelant, en plus ramassé, celle des yatagans albanais ou des flissahs kabyles;

2 , soie : dans le prolongement du dos de la lame et non au milieu de celle-ci, ainsi qu'il est en usage dans la plupart des armes;

3, poignée : légèrement courbée, terminée par un pommeau représentant une tête d'animal;

4 , garde : consistant uniquement en une traverse ou croisière rectangulaire;

5 , tenon ou arête : à sa partie inférieure, la poignée s'élargit jusqu'à s'étaler sur toute la longueur de la traverse. A l'extrémité de cette dernière, opposée à la soie, la poignée forme une excroissance ou tenon en arête, sorte d'embryon de branche de garde destiné à retenir la main. 


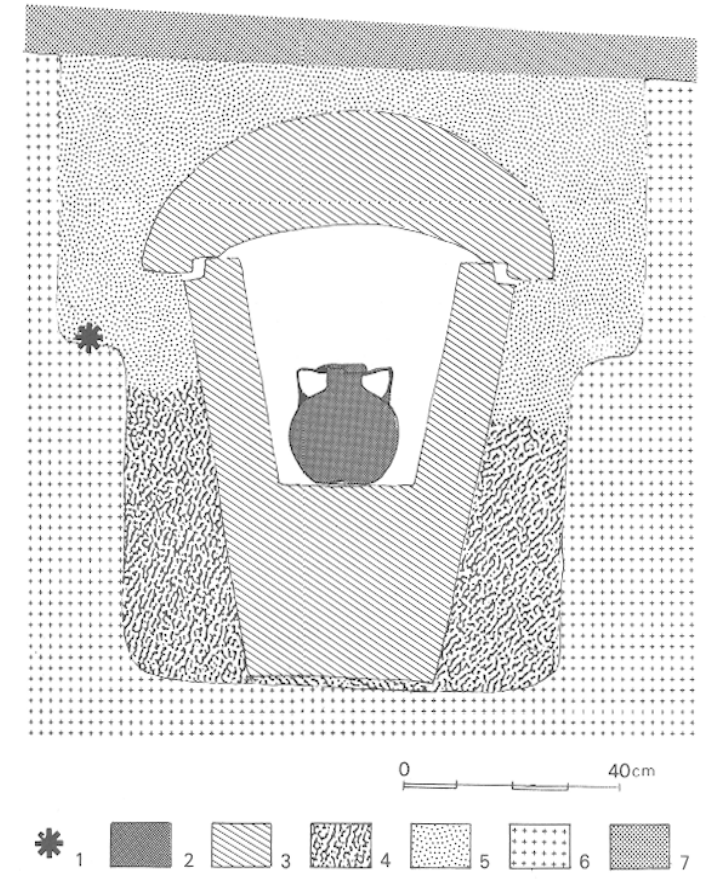

Fig. 1 - Coupe schématique d'une sépulture gallo-romaine avec coffre funéraire : Réserva, commune de Saint-MartinTerressus (d'après J. Perrier).

1 , coutelas posé sur une banquette $; 2$, urne en verre 3 , coffre funéraire ; 4 , résidu de la crémation; 5 , terre de comblement (stérile); 6 , roche naturelle; 7 , terre arable.

Ces critères n'ont pas été retenus car il importait d'affiner la typologie de ces armes, et éventuellement de définir leur fonction. Pour cela, tous les coutelas et les poignards ont été classés selon les critères suivants :

1 , longueur de la lame inférieure à $0,20 \mathrm{~m}$;

2 , longueur de la lame supérieure à $0,20 \mathrm{~m}$;

3 , lame de forme ondulée;

4, lame de forme triangulaire;

5 , soie située dans l'axe médian de la lame;

6 , soie située dans le prolongement du dos de la lame;

7, poignée en bronze coulée d'une seule pièce;

8 , fourreau en bronze;

9, présence seule de la garde en bronze;

10 , garde et bouterolle en bronze, présumant un fourreau en matière périssable.

Cette liste de caractères peut sembler limitative. Il convenait toutefois de ne pas retenir ceux qui, en raison des lacunes de notre documentation, obligeaient à éliminer un trop grand nombre d'objets.

Le tableau disjonctif élaboré selon ces critères a permis d'obtenir une classification hiérarchique as-

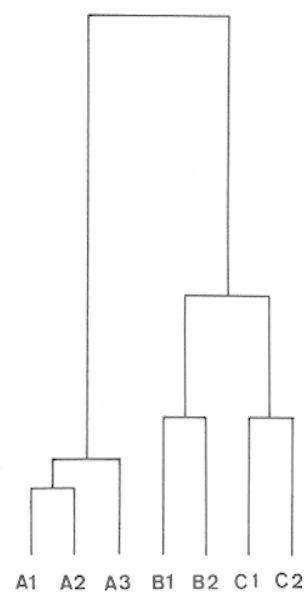

Fig. 2 - Partie supérieure de l'arbre hiérarchique.

cendante en utilisant la méthode du lien moyen sur la distance de Jaccard. Celle-ci est en effet la mieux adaptée au traitement des variables qualitatives (Roux, 1985, p. 33).

Le nœud le plus élevé de l'arbre hiérarchique (fig. 2) individualise les objets miniaturisés. En effet, plusieurs caractères (poignée moulée, fourreau en bronze coulé d'une seule pièce ...) en font des objets à part. Le nœud suivant permet de distinguer deux classes dans les objets miniaturisés, en raison de la position de la soie. Les autres nœuds se situent pratiquement à un même niveau, ce qui indique l'existence de trois familles :

- A. Objets dont la longueur de la lamè dépasse $0,20 \mathrm{~m}$ et qui peuvent par conséquent être des coutelas (fig. 3).

- B. Petits poignards avec une soie placée dans l'axe médian de la lame (fig. 4).

- C. Petits coutelas dont la soie se situe dans l'axe du dos de la lame (fig. 5).

Cette classification met en évidence trois familles que l'on soupçonnait déjà au premier examen du matériel. Elle permet toutefois d'affiner la typologie suivant une troncature de l'arbre hiérarchique effectuée à un niveau inférieur.

\section{TYPE Al}

Il comprend les coutelas à lame ondulée avec fourreau en matière périssable dont ne subsistent qu'une bouterolle et une entrée en feuille de bronze. La garde en bronze subsiste également. Pour les deux individus de ce type, la soie s'interrompt à faible distance du talon de la lame. Le coutelas 

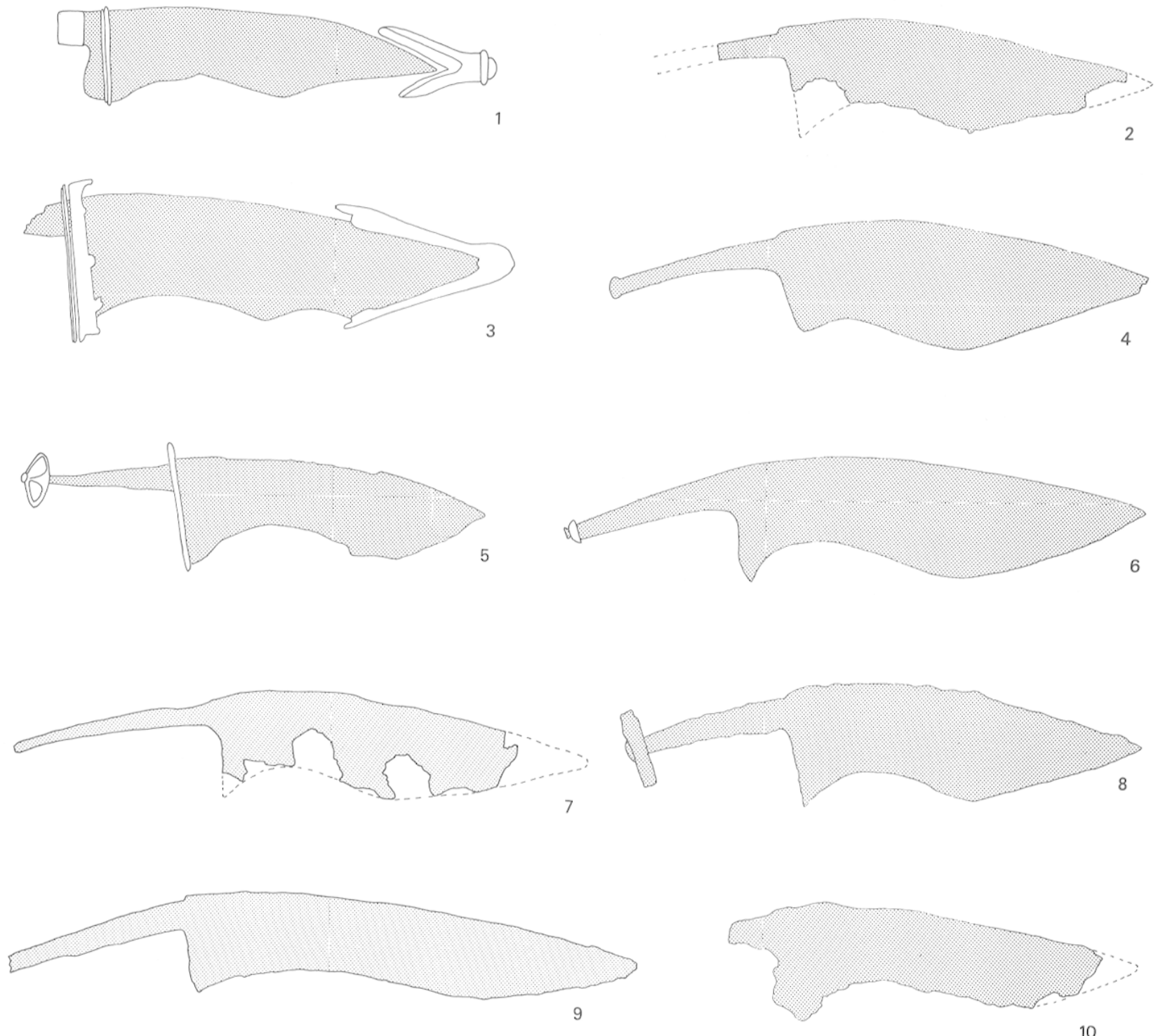

10
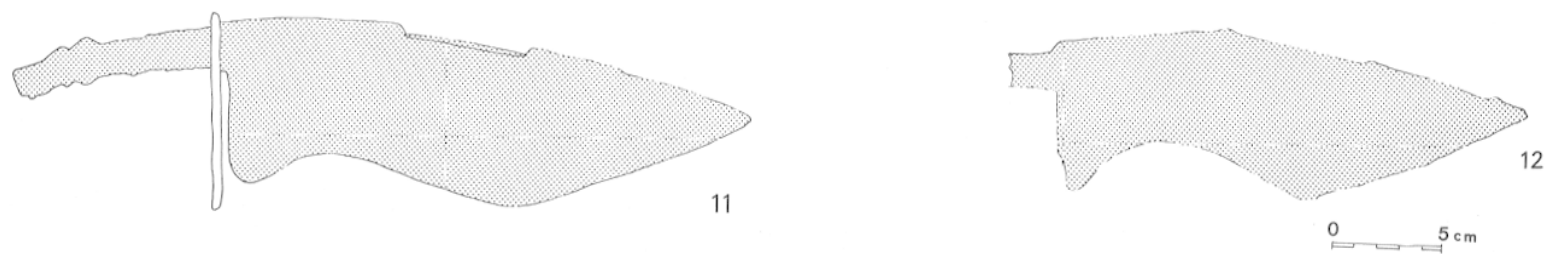

Fig. 3 - Les coutelas de type A 1, A 2 et A 3. Corrèze: 1, Argentat (inv. 2); 2, Eyrein (inv. 3). Creuse : 3, Azérables (inv. 10) 4, Lépinas (inv. 16); 5, Méasnes (inv. 19); 6, Savennes (inv. 31); 7, Saint-Christophe (inv. 21); 8, Saint-Martin-Sainte-Catherine (Le Theil) (inv. 29); 9, Saint-Priest (inv. 30). Hautr-Vienne: 10, Châteauponsac (inv. 34); 11, Saint-Laurent-les-Églises (inv. 39); 12, Saint-Martin-Terressus (inv. 40).

d'Argentat comporte, à l'extrémité de la soie, une bague en bronze qui suggère l'existence d'une poignée en métal aujourd'hui disparue, fixée en force sur le moignon de la soie. Ces coutelas rappellent les petits poignards du type C2 ainsi que les armes figurées sur les arcs de triomphe d'Orange ou de Carpentras. Ils correspondent également aux coutelas connus en Côte-d'Or, munis d'une poignée en bronze dont le pommeau est une tête d'animal (Reynier, 1959). 


\section{TyPE A2}

Ce sont des coutelas à lame ondulée sans aucun attribut rappelant un fourreau ou une garde. La soie maintenait une poignée en matière périssable arrêtée, vers l'avant, par le talon de la lame ct un cran faisant office de butee, à l'extrémité du dos de la lame. Elle était maintenue par une rondelle en fer ou en bronze, fixée par martellement de l'extrémité de la soie. La longueur de la lame est en principe supérieure à celle des coutelas des types A1 et A3.

\section{TYPE A3}

Deux coutelas appartiennent à ce type. Ce sont des formes intermédiaires entre les types Al et A2. Ils possèdent une garde en bronze. L'aspect très corrodé de la surface de la lame suggère la présence d'un fourreau en fer plutôt qu'une desquamation de la lame due uniquement à l'oxydation. Bien que la rondelle de cuivre de l'exemplaire de Méasnes (Creuse) rappelle un pommeau, la poignée des coutelas de ce type était en matière périssable.
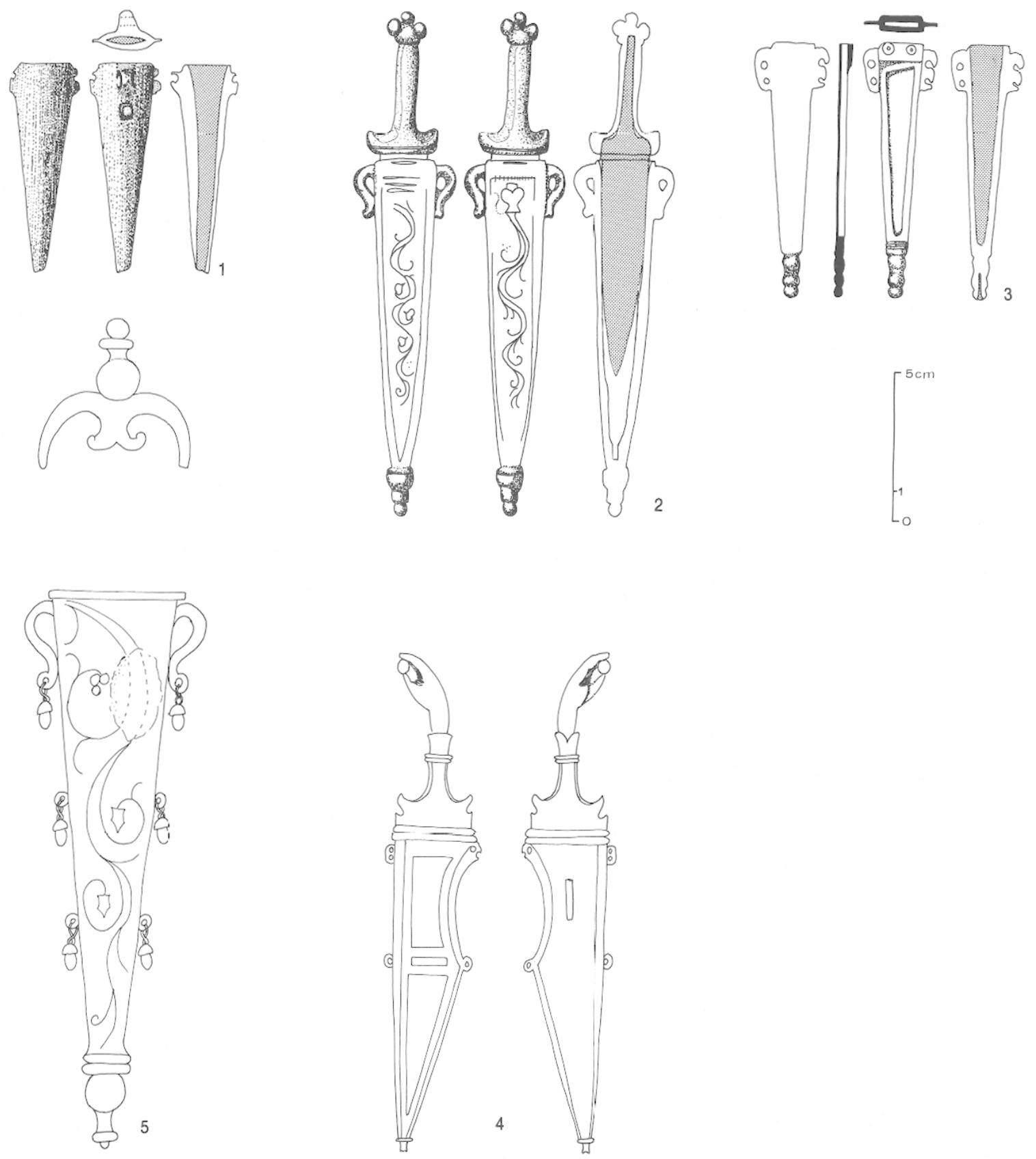

Fig. 4 - Poignards de type B I et B 2. Cireuse : 1, Ahun (inv. 8) ; 2, Saint-IIilaire-le-Chäteau (inv. 25) ; 3, Saint-Maixant (inv. 27); 4, Saint-Martin-Sainte-Catherine (La Texonnière) (inv. 28). Haute-Vifnnf: :5, Limoges (Usine à gaz) (inv. 37). 


\section{TYPE Bl}

Seul l'exemplaire de La Texonnière, commune de Saint-Martin-Sainte-Catherine (Creuse), appartient à ce type. Sa lame ondulée ressemble à celle des coutelas mais sa poignée, placée dans l'axe médian de la lame, le rapproche des poignards. La poignée, constituée par une main qui tient une petite boule entre le pouce et l'index, diffère des têtes animales des coutelas miniaturisés, sans pour autant se rapprocher des pommeaux symétriques des poignards.

\section{TYPE B2}

Les poignards de ce type n'entrent pas dans la catégorie des "glaives à lame ondulée" du Commandant Martignon. Ce sont des poignards caractérisés par une poignée placée dans l'axe médian de la lame triangulaire et symétrique par rapport à son axe longitudinal. La poignée droite se termine par un pommeau symétrique. Seules les dimensions réduites de la lame les rapprochent des coutelas miniaturisés. Les fourreaux d'Ahun et de Saint-Maixant (Creuse) doivent appartenir à des poignards de ce type.
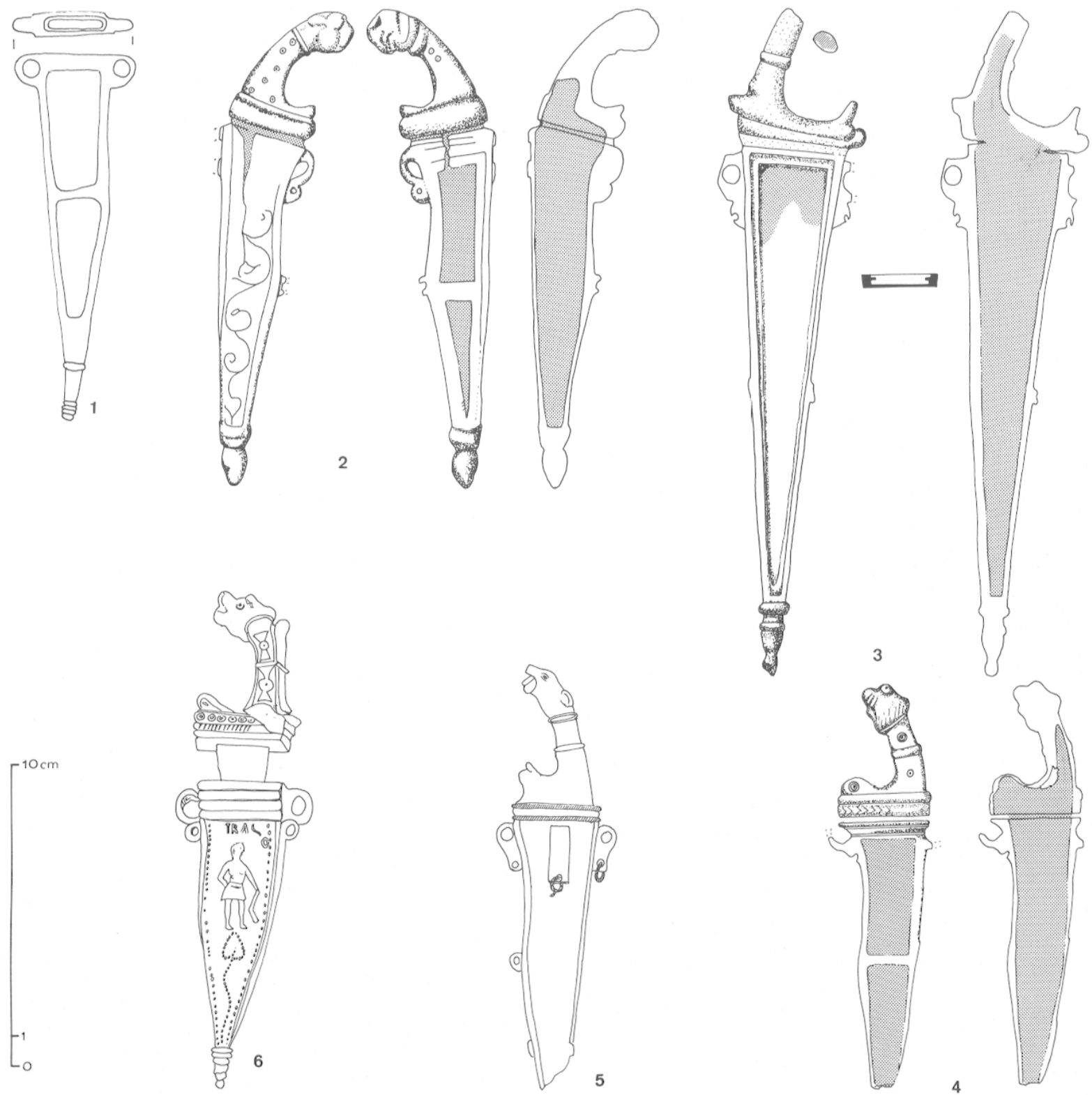

Fig. 5 - Coutelas miniaturisés de type C 1 et C 2. Corrèze : 1, Affieux (inv. 1). Creuse : 2, Le Grand-Bourg (inv. 13); 3, Janaillat (inv. 14). Haute-Vienne: 4, Flavignac (inv. 35); 5, Limoges (Manège de Cavalerie) (inv. 36); 6, Razès (inv. 38). 


\section{TYPE C1}

Il ne comprend qu'un exemplaire et diffère des coutelas miniaturisés en raison de la forme triangulaire de sa lame. Il s'en rapproche toutefois par l'emplacement de sa poignée et par' la dissymétrie de la lame. (Ce dernier caractère n'a toutefois pas été pris en compte pour le calcul de la classification.)

\section{TYPE C2}

Ce sont les coutelas miniaturisés à lame avec une faible concavité qui rappellent le mieux certaines armes représentées sur les arcs de triomphe d'Orange et de Carpentras. La lame ondulée s'insère dans un fourreau dont l'armature, coulée d'une seule pièce, comporte des rainures permettant d'encastrer des plaques qui ont souvent disparu. Seul un exemplaire a conservé ses deux plaques: l'une possède un dispositif de fixation, l'autre un décor gravé. La poignée, fixée sur la lame par coulée se trouve dans le prolongement du dos de la lame. Généralement ornée, elle se terminne souvent par une tête animale qui constitue le pommeau.

\section{TECHNOLOGIE}

Grâce à l'obligeance du $\mathrm{D}^{\mathrm{r}}$ Maraillat, radiologue, quelques objets ont pu être radiographiés, ce qui a permis de préciser leur morphologie interne ${ }^{1}$. L'examen direct et radiographique des poignards et des coutelas miniaturisés permet de dresser un schéma de la technologie employée dans leur fabrication.

Les poignées de bronze des coutelas miniaturisés dont la soie se trouve dans le prolongement du dos de la lame (Type C1 et C2) sont coulées directement sur la soie en fer. On retrouve ici une tradition de fabrication connue dès le début du Premier Age du Fer. Par contre, le manche du poignard de SaintHilaire-le-Château (Creuse) qui appartient au type $\mathrm{B} 2$ est coulé à part puis assemblé sans rivets sur la soie.

Les coutelas de type $\mathrm{A} 2$ et $\mathrm{A} 3$, forgés d'une seule pièce, ont le talon de la lame bien marqué complété par un cran qui apparaît nettement à l'extrémité du dos de la lame, dans le prolongement du talon. Ces deux particularités pouvaient servir de

1 Les radiographies ont été effectuées à l'hôpital de Guéret sur négatifs CRONEX Médicis MRF 31 (Dupont de Nemours) à $147 \mathrm{kv}, 400 \mathrm{mAS}$ et MAMORAY MR3 NIF (AgfaGevaert) à $140 \mathrm{kv}, 30 \mathrm{mAS}$ et $150 \mathrm{kv}$ à $50 \mathrm{mAS}$ et $145 \mathrm{kv}$ entre $60 \mathrm{mAS}$ et $300 \mathrm{mAS}$. retenue et de butée à une simple poignée (Type A2) ou à une garde en bronze rajoutée (Type A3). Dans ce cas, la garde n'est qu'une simple barrette de bronze ajourée en son centre puis glissée sur la soie. Lorsque les soies sont complètes, le pommeau se compose d'une barrette en fer ou d'une virole en bronze retenues au sommet de la poignée par rivetage à chaud de l'extrémité proximale de la soie.

Les fourreaux en bronze des poignards ou coutelas miniaturisés sont coulés d'une seule pièce, qu'ils soient ajourés ou non. Bélières et bouterolles, obtenues directement par coulée n'ont pas été rajoutées. Seule l'extrémité de la bouterolle de SaintMaixant est creuse.

\section{LES DÉCORS}

En Limousin, seuls les coutelas et les poignards miniaturisés possèdent un décor sur la poignée ou sur le fourreau.

Un pommeau en ronde bosse qui représente généralement une tête d'animal, le plus souvent un lion, prolonge la poignée des coutelas miniaturisés. Le motif peut toutefois être différent comme par exemple la main qui tient une petite boule du coutelas miniaturisé de La Texonnière, commune de Saint-Martin-Sainte-Catherine (Creuse). Ces motifs obtenus par coulée sont parfois réhaussés par des incisions gravées au burin. C'est par ce moyen qu'est obtenu le rendu de la crinière du coutelas miniaturisé de Flavignac (Haute-Vienne).

Le pommeau des poignards miniaturisés, très différent, offre toujours un motif géométrique, symétrique par rapport à l'axe longitudinal de l'arme.

Les motifs utilisés pour former le pommeau sont présents non seulement sur les coutelas figurés sur les monuments du Sud de la Gaule mais aussi sur des armes de Bourgogne (Reyniers, 1959). Ils sont également utilisés pour orner des manches de clé. Bien que, dans ce cas, le fauve soit souvent représenté en entier ou presque (Espérandieu, 1959, $\mathrm{n}^{\text {os }} 163$ à 166; Faider-Feytmans, 1957, nos 253 à 255 ), il arrive que seul un protomé de lion orne l'extrémité de la poignée (Faider-Feytmans, 1957, $\mathrm{n}^{\text {os }} 247$ et 248 ; Espérandieu, 1959 , nos 166 et 167 ; Majurel, $1964, \mathrm{n}^{\text {os }} 1$ et 2 ).

La main tenant une boule entre le pouce et l'index est également un motif qui orne l'extrémité des manches de clé (Lebel, 1959 a, pl. LXXIX, n 6 ; Armand-Caillat, 1957). Ce motif a été étudié par P. Lebel (1961). 
Le corps de la poignée des coutelas miniaturisés s'orne fréquemment de cercles pointés ou de cercles concentriques obtenus par estampage. Ils sont disposés au hasard, sauf sur l'exemplaire de Razès (Haute-Vienne) où ils sont alignés sur la garde.

Pour certains auteurs, ce motif apparaît dès la préhistoire (Devauges, 1981a). Il est en tout cas connu sur de la céramique du Bronze final en Franche-Comté (Collectif, 1981) et très répandu au Premier Age du Fer sur les brassards, bracelets ou les plaques de ceinture de la forêt de Haguenau (Schaeffer, 1930). Au Second Age du Fer, ce motif orne les torques à tampons en Champagne (BretzMahler, 1971, pl. 43), l'arc de fibules en Europe Centrale (Kruta, 1971, p. 22-23 et pl. 10 à 17). Quatre-vingt-neuf fibules de Douchcov sont ornées de cercles, simples ou doubles qui représentent le principal décor. Ce motif est également fréquent sur la céramique, par exemple en Auvergne (Chopelin, 1973). Un couteau ou coutelas de La Tène à lame triangulaire mis au jour à Kernavest en Quiberon (Morbihan) possède un fourreau richement orné. Des lignes de cercles occulés alternent avec les lignes de triangles ou de motifs curvilignes (Giot, 1969, p. 62$63)$.

L'ornementation des fourreaux des coutelas miniaturisés se limite à une seule plaque. L'autre possède seulement un pontet de fixation. Seules deux plaques ornées de coutelas miniaturisés nous sont connues. Ainsi les deux plaques du fourreau de Leyrit, commune d'Affieux (Corrèze), avaient disparu lors de la découverte. Ces plaques étaient en effet simplement serties sur l'armature.

La plaque du fourreau de Razès (Haute-Vienne) est grossièrement triangulaire. Les côtés sont soulignés d'une juxtaposition de points. La représentation d'une feuille de lierre et de sa tige sur la pointe distale du fourreau relève de la même technique. Un personnage debout, torse et jambes nus, tenant dans sa main un objet indéterminé, est obtenu par des traits incisés. Il est surmonté d'une inscription qui a été lue IRAC ou TRAS. La plaque de Grand-Bourg (Creuse) s'orne d'un rinceau qui se termine par une feuille de lierre.

Ce sont des rinceaux similaires que l'on trouve sur chacune des faces des poignards miniaturisés de l'Usine à Gaz à Limoges et de Saint-Hilaire-leChâteau (Creuse). En outre, le poignard de Limoges portait six glands suspendus aux anneaux latéraux par de petites chainettes.

\section{COMPARAISONS}

La figuration de plusieurs exemplaires de coutelas ou de glaives à lame ondulée sur les trophées des ares de triomphe de Narbonnaise permet à $P$. Couissin (1924, p. 35) d'affirmer que : "l'épée des trophées est essentiellement le sabre ondulé».

L'exemplaire le plus proche des armes à lame ondulée figure sur la face latérale e. de l'arc de triomphe de Carpentras (Espérandieu, 1907, $\mathrm{n}^{\circ} 243$ ). Il apparaît, suspendu à un baudrier, aux pieds d'un captif enchaîné. Le dessin de Caristie, reproduit par Espérandieu, laisse entrevoir un autre de ces glaives sur la face o. du même monument. Attaché à un ceinturon, il possède une poignée terminée par une tête de lion.

Deux poignées au pommeau en forme de tête d'oiseau figurent sur l'arc de triomphe d'Orange. La forme d'un fourreau suggère que leur lame ètait ondulée (Amy, 1962, p. 85 et pl. 49).

Deux exemplaires sculptés sur la mouluration de l'imposte de l'arc de triomphe de Glanum sont munis d'une poignée simple. Le fourreau n'est pas représenté ce qui laisse nettement apparaître la concavité du tranchant de la lame (Rolland, 1977, fig. 6, p. 30 et pl. $21, \mathrm{n}^{\text {os }} 4$ et 7 ).

Un bas-relief de Narbonne montre, sur la droite, un glaive dans son fourreau, fixé à un ceinturon. La poignée se termine par une tête d'oiseau, comme à Orange (Espérandieu, 1907, $\mathrm{n}^{\circ} 691$ ).

A Vienne, un glaive visible sur un fragment de bas-relief possède une tête d'animal en guise de pommeau (Espérandieu, 1907, no 375 ).

P. Couissin (1923, p. 32-36 et 1927, p. 171-174), après H. Breuil (1917), étudie les sabres à lame ondulée et, entre autres, les coutelas à fourreau courbe et ceux à fourreau droit. Certaines des armes citées dans ces deux dernières séries ne possèdent pas, du moins si l'on s'en tient au dessin du fourreau, la concavité caractéristique du tranchant de la lame. Elles s'en rapprochent toutefois par l'emplacement de la soie et la forme de la poignée. L'exemplaire figuré sur la face e. de l'arc de triomphe de Carpentras est classé, à tort, parmi les coutelas à fourreau courbe (Couissin, 1923, p. 36 et fig. 2, no 1).

Quant aux coutelas à lame ondulée mis au jour fortuitement ou en cours de fouille, les exemplaires que nous avons pu recenser sont extrêmement rares et souvent assez différents de ceux du Limousin. 
Mont-Beuvray (Saône-et-Loire). Poignée d'un petit coutelas dont le pommeau représente une tête de cheval (Déchelette, 1904, fig. $\mathrm{n}^{\circ} 16$ ).

Couternon (Côte-d'Or). Musée de Saint-Germain-enLaye (Inv. 65 451). Coutelas à lame ondulée long de $0,29 \mathrm{~m}$. La poignée en bronze se termine par un pommeau en tête de lion (Reyniers, 1959).

Villargoix (Côte-d'Or). Musée archéologique de Dijon (Inv. $\mathrm{n}^{\circ} 503$ ). Long de $0,44 \mathrm{~m}$, ce coutelas possède une lame identique à celui du Boisseau, commune de Saint-Martin-Sainte-Catherine (Creuse). La poignée en bois est encadrée par une garde en bronze, ornée d'un petit lion et par un pommeau représentant un mufle de lion (Lebel, 1959 b, fig. 73).

Grange-le-Bourg (Haute-Saône). Coutelas à lame très oxydée et poignée rectiligne, en bronze, munie d'un pommeau en tête de lion (Long. 0,43 $\mathrm{m}$ dont 0,28 pour la lame) (Reyniers, 1959).

Dampierre-les-Langres (Haute-Marne). Musée (Inv. $\mathrm{n}^{\circ}$ 231). Coutelas long de $0,40 \mathrm{~m}$ à lame à peine échancrée. la poignée en bronze se termine par un pommeau en tête de lion (Reyniers, 1959).

Chalon-sur-Saône (Saône-et-Loire). Musée (Inv. $\mathrm{n}^{\circ} 391$ ). Long de $0,325 \mathrm{~m}$, sa poignée de bronze se termine par un mufle qui n'est peut-être pas celui d'un lion (Reyniers, 1959).

Musée de Besançon (Doubs). Coutelas à lame nettement ondulée longue de $0,242 \mathrm{~m}$. La soie, longue de $0,14 \mathrm{~m}$, ne prolonge pas le dos de la lame mais laisse un cran plus important que ceux des exemplaires limousins (environ un cinquième de la hauteur du talon de la lame) (Inv. A 395). Le même musée possède des coutelas à poignée de bronze, parfois évidée, placée dans le prolongement du dos de la lame. L'un d'eux (Inv. A 394) possède une lame au tranchant ondulé qui mesure $0,255 \mathrm{~m}$ avec une poignće de $0,10 \mathrm{~m}$. La provenance de ces objets n'est pas connue. (Renseignement: Ph. Lagrange, conservateur).

Saint-Just (Charente-Maritime). Coutelas miniaturisé long de $0,079 \mathrm{~m}$ dont la lame est encore engagée dans son fourreau en tôle de bronze. La forme de la lame ne peut être précisée (David, 1974 - étude de J. Gomez de Soto, p. 119-120). Coutelas à lame échancrée, long de $0,26 \mathrm{~m}$, mis au jour dans un puits à Pépiron (Eygun, 1965).

Faye-l'Abbesse (Deux-Sèvres). Musée du Pilori à Niort (Inv. 502). Grand coutelas en fer du type A2.
Il se trouvait associé, dans les réserves du musée, à des armes celtiques (Gendron, 1986, p. 91, fig. 5).

Germond (Deux-Sèvres). Musée du Pilori à Niort. Coutelas miniaturisé dans son fourreau de bronze. Le pommeau est un sabot de cheval (Renseignement : J. Gomez-de-Soto et Gendron, 1986, nº 10).

Mâlain (Côte-d'Or). Coutelas long de 0,21 m, entièrement en bronze, avec une lame légèrement échancrée et une soie rectiligne dont l'extrémité semble brisée (Devauges, 1981 b, p. 422, fig. 8).

Bâle (Suisse). Poignée de bronze terminée par une tête d'oiseau, probablement un rapace, qui tient une boule dans son bec. La lame de cet objet, très oxydée, ne peut être décrite (Fellmann, 1955, pl. 26, $\left.\mathrm{n}^{\circ} 3\right)$.

L'Hospitalet-du-Larzac (Aveyron). Un couteau découvert dans une sépulture de l'époque de Claude possède une lame qui, sans être franchement échancrée, présente un tranchant concave (Lequément, 1983).

Aislingen (Allemagne). Coutelas dont la lame, longue de $0,24 \mathrm{~m}$, est nettement concave. Toutefois, la soie plate prend naissance au milieu du talon de la lame (Ulbert, 1959, pl. 28, n 18).

Enfin, un type de fibule, connu en deux exemplaires, reproduit un coutelas à lame ondulée dans un fourreau (de cuir?) décoré de ponctuations (Feugère, 1985, fig. 56, type $28 \mathrm{G}$ ). La datation proposée pour ces objets couvre la période d'Hadrien aux Antonins.

\section{LES ARMES DANS LES SEPPULTURES}

Coutelas et poignards, quelles que soient leurs dimensions, ne sont pas les seules armes rencontrées dans les sépultures à incinération du Limousin. Un invenlaire, probablement incomplet, révèle la présence d'au moins douze tombes avec épée, neuf tombes avec lance, une tombe avec un trident en fer. Ces chiffres, ajoutés aux quarante exemplaires répertoriés ici, donnent un total d'une soixantaine de sépultures avec armes.

Pendant longtemps, le dépôt d'armes dans les sépultures gallo-romaines était considéré comme une pratique étrangère et plus particulièrement germanique. H. Schonberger (1953) insista sur le fait que les sépultures gallo-romaines du Haut-Empire renfermaient des armes tout en précisant que ce rite n'était ni romain, ni italique. 
Par la suite, Van Doorselear (1965), a repris le problème et dressé un inventaire qui montre que le dépôt d'armes dans les sépultures gallo-romaines était encore plus répandu que le soupçonnait Schonberger : il a dénombré une centaine de tombes avec armes qui se répartissent dans 91 nécropoles sur 2600 connues. En étudiant la fréquence des sépultures avec armes à l'intérieur des nécropoles, on remarque qu'elle est en réalité très faible. A Blicquy (Belgique) où la proportion de tombes avec armes est considérée comme très importante, dix tombes sur 407 en renfermaient. Encore s'agit-il d'un pilum, d'une pointe de lance et de huit couteaux triangulaires à douille (De Laet, 1972, p. 28-30).

En Bretagne, des armes sont également connues dans des sépultures du Haut-Empire. L'inventaire qu'en dresse P. Galliou (1981, p. 146-147) se limite à sept dépôts funéraires qui ont livré des épées ou glaives, dagues, couteaux, fourche, hache et bouclier.

En Limousin, la fréquence des tombes avec armes nous semble supérieure. Sans décompter les haches ou les fauchards considérés commes outils, ni les couteaux triangulaires, canifs ou couteaux pliants qui sont pour nous des objets domestiques ou usuels (Lintz, 1987), le nombre de sépultures avec arme doit approcher 2 à $3 \%$. Cette proportion est donc très importante comparée aux nombreuses nécropoles de Gaule qui n'en ont livré aucune, que ce soit à Poitiers (Eygun, 1933), aux Bolards (Nuits-SaintGeorges, Côte-d'Or) (Planson, 1982), à Apt ou à Cavaillon (Vaucluse) (Dumoulin, 1964 et 1965) ou encore à Cabasse dans le Var (Bérard, 1961).

On ne peut guère évoquer la présence de tombes de militaires pour expliquer le dépôt d'armes dans les sépultures $d u \mathrm{II}^{\mathrm{e}}$ s. en Limousin. Par contre, cette époque correspond à une période où la chasse connaît un regain de faveur (Aymard, 1951, p. 157). A Rome, les empereurs eux-mêmes s'y consacrent. La chasse tenait également une place importante chez les Celtes, en particulier dans l'aristocratie (Aymard, 1951, p. 65-66). Or, il apparaît que les sépultures avec armes se trouvent parmi les plus riches du Limousin (Mennessier, 1973, p. 90). Sur dix-neuf sépultures suffisamment bien décrites qui ont livré des coutelas, quinze possèdent une urne en verre, trois une urne en terre cuite et, dans un seul cas, la tombe ne comporte pas d'urne. En outre, elles renferment souvent un mobilier abondant parmi lequel se retrouvent des objets en or comme par exemple à Chambonchard et à Saint-Christophe (Creuse) ou à Saint-Laurent-les-Églises (Haute-Vienne).
Les sépultures avec coutelas sont, par conséquent, des sépultures riches dont certaines recèlent également d'autres armes : quatre pointes de javelot à Lépinas (Creuse), une épée et une pointe de lance à Saint-Laurent-les-Églises (Haute-Vienne), un fer de lance à Thouron (Haute-Vienne).

Bien que les coutelas soient représentés sur les trophées de certains arcs de triomphe, ils ne semblent pas figurer parmi l'armement celte tel que nous le font connaître les textes antiques (Couissin, 1927, p. 171-172; Deyber, 1986). L'hypothèse d'armes de chasse parait plus vraisemblable et n'exclut d'ailleurs pas leur utilisation pour le combat. Ainsi, Tacite (Annales, III, 43) décrit l'armement des troupes de Sacrovir: "Ils étaient quarante mille, dont le cinquième étail armé comme nos légionnaires. Le reste avait des épieux, des coutelas et toutes les autres armes qu'on emploie à la chasse" (traduction Lerat, 1977, p. 143).

Un coutelas, morphologiquement assez proche de ceux à lame ondulée, est d'ailleurs représenté sur un bas-relief conservé au musée du Puy, parmi une panoplie d'armes de chasse (Espérandieu, II, 1909). Les armes retrouvées dans les sépultures galloromaines du Limousin seraient donc des armes de chasse qui accompagnaient leur propriétaire dans sa dernière demeure.

Ces observations nous conduisent à réviser les hypothèses émises à la suite de la découverte d'une importante sépulture à la Bussière-Étable, commune de Châteauponsac (Haute-Vienne). Mis au jour en décembre 1946, le tombeau comprenait une fosse de 1,10 à $1,20 \mathrm{~m}$ de diamètre et $0,75 \mathrm{~m}$ de profondeur recouverte par un massif de maçonnerie long de $13 \mathrm{~m}$ sur $10 \mathrm{~m}$ de large. La fosse a livré un matériel de même nature que celui rencontré dans les dépôts funéraires mais en quantité plus abondante (Baubérot, 1950; Salin, 1951). Outre la céramique et la verrerie, cette tombe a livré un abondant matériel métallique comprenant :

- des objets usuels en fer : pioche, pelle à long manche, probablement une pelle de foyer, des pinces de forgeron et un trépied;

- des armes : une épée courte, deux fers de lance, quatre lances à crochets, trois fauchards et une hache à double tranchant;

- huit bandages de roues de char et des ornements de char en bronze d'une extrême variété.

Recherchant des comparaisons dans l'armement militaire, E. Salin a été conduit à rapprocher la sépulture de la Bussière-Étable des tombes royales 
de Russie méridionale. Pour expliquer sa présence sous les Antonins, il émet l'hypothèse de la tombe d'un prince barbare compromis dans les révoltes de 117-118 et exilé (Salin, 1951, p. 113-115). A l'époque, il ètait en effet admis que seules des sépultures de barbares pouvaient enfermer des armes.

Notre hypothèse est différente. En effet, les armes mises au jour à la Bussière-Étable ne diffèrent pas de celles retrouvées dans d'autres sépultures. Ainsi une lance à crochets est présente dans la tombe de Thouron (Haute-Vienne), associée à un coutelas. Cette pointe de lance, ou pointe d'épieu à barbelures est d'ailleurs une arme de chasse décrite par Xénophon puis par plusieurs auteurs; sous l'Empire, son utilisation est attestée pour la chasse au sanglier (Aymard, 1951, p. 310-313). Une hache à double tranchant est figurée sur la face e. de l'arc de triomphe de Carpentras où elle fait pendant à un coutelas. Par ailleurs, le rapport de gendarmerie relatant la découverte des sépultures du Leyrit, commune d'Affieux (Corrèze) mentionne une hache double. Nous n'avons pas considéré les fauchards comme des armes mais ils se rencontrent également dans les sépultures. Ainsi la sépulture du Boisseau, commune de Saint-Martin-Sainte-Catherine (Creuse), outre le coutelas, a livré un fauchard et une hache miniaturisés.

Par conséquent, cette tombe de la BussièreÉtable pourrait très bien être celle d'un riche propriétaire gallo-romain souhaitant que tout son attirail de chasse l'accompagne dans l'autre monde. Le testament du Lingon (C.I.L., XIII, 5709) ne peut que confirmer une telle hypothèse. De son vivant, le testateur a fait construire un important monument funéraire. Dans ses instructions, il précise : «Je veux que tout mon attirail que je me suis procuré pour chasser et prendre les oiseaux soit brûlé avec moi : épieux, glaives, coutelas, filets, pièges, lacets, flèches, tentes, épouvantails, mes baignoires, mes litières, ma chaise à porteur et tout l'équipement et l'attirail en rapport avec cette occupation ..." (traduction Lerat, 1977, p. 298).

\section{OBJETS MINIATURISÉS}

Outre les coutelas et les poignards miniaturisés, d'autres objets de dimensions réduites se rencontrent parmi le mobilier des sépultures gallo-romaines du Limousin (Lintz, 1982). Dans d'autres régions, des nécropoles ou des sanctuaires ont livré de tels objets. Si, par exemple, l'interprétation comme ex-voto des armes miniatures mises au jour au cours de la fouille du sanctuaires de Flavier, commune de Mouzon dans les Ardennes (Tisserand, 1980) ne fait aucun doute, il n'en est pas toujours de même. En effet, les reproductions d'outils en fer (pioches, haches, herminettes, fauchards...) sonl généralement considérées comme des jouets d'enfants (Janicaud, 1943, p. 459). A Blicquy, des assiettes de très faibles dimensions servaient de couvercles à des urnes mais des chenets miniatures en terre cuite représentaient le culte du foyer en remplaçant les véritables chenets en fer (De Laet, 1972, p. 26-28 et 32). De nombreuses tombes du Limousin ont livré de petites céramiques qui, en raison de leurs caractères, ne pouvaient avoir aucune utilité ménagère. Contrairement aux céramiques normales, elles n'étaient d'ailleurs pas brisées sur le bûcher d'incinération. Par exemple, une sépulture mise au jour à Aubusson a livré seize céramiques miniaturisées (assiettes, jattes, tripodes, pots, bouteilles) trouvées intactes et sept céramiques de dimensions normales brisées sur le bûcher (Lintz, 1979). Les petites céramiques, mal façonnées et mal cuites, présentaient des fentes qui s'étaient formées au séchage ou même des trous causés par de gros cristaux de quartz ou de feldspath entraînés lors du tournage. De toute évidence, elles étaient fabriquées pour être placées dans la tombe. Dans d'autres cas, la fabrication de céramiques miniaturisées était au contraire soignée (Perrier, 1970).

Tels qu'ils se présentent, les poignards ou les coutelas miniaturisés ne peuvent avoir aucune utilité fonctionnelle. La poignée, en particulier, est insaisissable. Ce sont, à notre avis, des ex-voto placés dans la tombe en remplacement des objets véritables, comme d'ailleurs les outils miniatures ou les chenets en terre cuite de Blicquy.

\section{DATATION ET ORIGINE}

Les sépultures du Limousin qui renferment des armes sont toutes datées du II ${ }^{\mathrm{e}}$ s. et plus particulièrement de la seconde moitié. Les monnaies qui leur sont associées portent les effigies de Trajan, Hadrien ou Antonin. La datation des quelques incinérations qui possèdent des monnaies plus anciennes doit être reportée à la même époque en raison de la céramique, souvent abondante, trouvée dans le résidu de la crémation.

L'arc de triomphe d'Orange édifié sous Tibère et ceux de Carpentras et de Glanum, de quelques années plus anciens, montrent que les armes de ce type existaient avant même le début de notre ère. 
L'exemplaire du musée de Niort (Deux-Sèvres), classé avec des armes celtiques découvertes à Fayel'Abbesse, est peut-être antérieur à la conquête : ce n'est toutefois pas une certitude car sa provenance n'est pas assurée.

Quoi qu'il en soit, les coutelas à lame ondulée sont connus dès le début de notre ère. Ils existent encore, miniaturisés ou non, à la fin du $\mathrm{II}^{\mathrm{e}} \mathrm{s}$. Il est même possible que la forme particulière de ces coutelas ait perduré. En effet, une sépulture mérovingienne découverte à Bourges a livré un coutelas à lame ondulée, posé sur le couvercle d'un cercueil en plomb, lui-même placé dans un sarcophage de pierre (Kersers, 1891). La soie conservait les traces d'une poignée en os; le fourreau, en bois et cuir, possédait une entrée en feuille de bronze ajourée.

L'origine de ces coutelas est délicate à cerner. Parfois identifiés à des armes ibériques (Breuil, 1917), ils semblent, en fait, communs à tous les peuples méditerranéens (Couissin, 1923, p. 35 et 1927, p. 171-174; Martignon, 1941, p. 420-421).

Notons que des coutelas à antennes et à lame légèrement ondulée sont connus en Suisse au Premier Age du Fer. Des cercles concentriques, motif très fréquent sur les coutelas miniatures du Limousin, ornent leur poignée (Drack, 1972/73, fig. 15). La lame ondulée se retrouve à Bologne au début de l'Age du Fer (Killian, 1970, fig. 3). Il faut observer également une légère ondulation du tranchant de la lame sur certains coutelas de la Marne au Deuxième Age du Fer (Déchelette, 1927, fig. 579, $\mathrm{n}^{\mathrm{o}} 1$; Guillaumet, 1975, $\mathrm{n}^{\circ} 103$ ). L'hypothèse de l'évolution d'un coutelas celtique n'est donc pas exclue.

Les coutelas à lame ondulée mis au jour dans les sépultures gallo-romaines à incinération du Limousin sont des armes de chasse gallo-romaines dont l'origine n'est pas clairement établie. Ils étaient connus dès le début de notre ère où ils ont pu compléter l'armement de gaulois révoltés. Ils paraissent fréquents $a u$ II $^{\mathrm{e}} \mathrm{s}$. à une époque où la chasse est devenue une occupation courante dans les classes aisées.

\section{Guy Lintz et Dominique Vuaillat}

N.B. - Nous remercions tous ceux qui nous ont aidés dans ce travail : $M^{\text {mes }}$ Doublard du Vignaud, Conservateur du Musée de Guéret et F. Trottignon, MM. D. Dussot, J. Gomez de Soto, $\mathrm{Ph}$. Lagrange, Conservateur de Musée à Besançon ainsi que $M^{\text {lle }}$ M.-C. Léonard et la Mairie de Flavignac. 


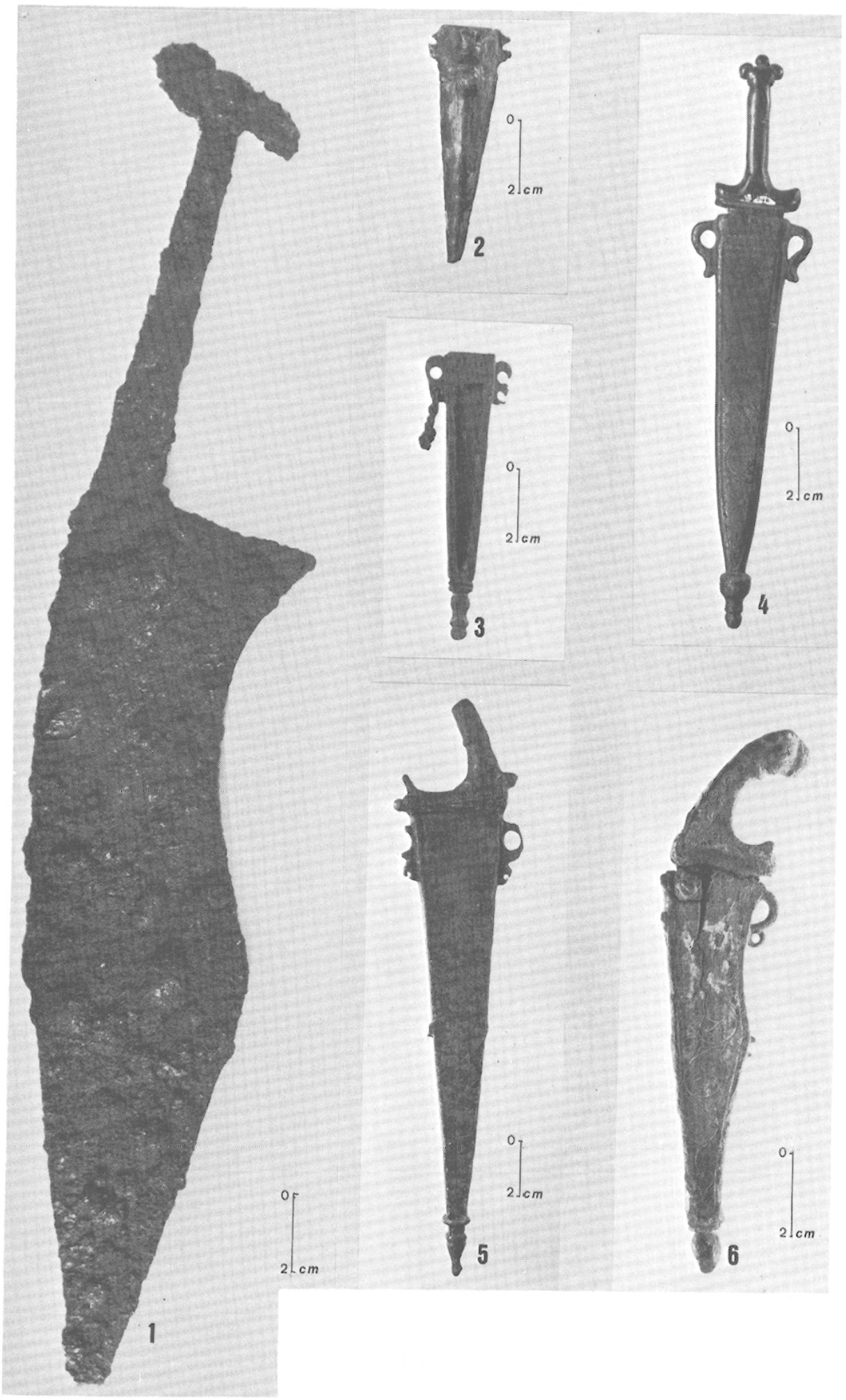

Fig. 6 - CReuse : 1, Saint-Martin-Sainte-Catherine (Le Theil) (inv. 29); 2, Ahun (inv. 8); 3, Saint-Maixant (inv. 27) ; 4, Saint-Hilaire-le-Château (inv. 25); 5, Janaillat (inv. 14); 6, Le Grand-Bourg (inv. 13). 


\section{INVENTAIRE DES DÉCOUVERTES}

L'inventaire prend en compte toutes les découvertes que nous avons retrouvées en bibliographie. Dans certains cas, les indications ne permettent pas de décrire les objets cités. Dans d'autres, l'enquête que nous avons conduite montre que le renseignement est erroné (voir par exemple Arrènes [ $\left.n^{\circ} 9\right]$ et Jarnages [ $\left.\mathbf{n}^{\circ} 15\right]$, Creuse).

Les points de découverte sont portés sur la carte (fig. 7 voir infra p. 185). Le numéro renvoie à la notice de l'inventaire. L'examen de la répartition des découvertes montre une concentration de coutelas et de poignards dans le n.-o. de la Creuse et le n.-e. de la Haute-Vienne (plateaux de la Marche). Cette abondance de trouvailles n'est pas directement corrélée à une forte concentration de sépultures avec coffres funéraires. En effet, une région comme la HauteCorrèze où les coffres funéraires sont également fréquents, n'a livré que peu de tombes avec poignards ou coutelas. Par contre, une liaison plus forte existe entre la répartition des tombes avec armes et une plus grande fréquence d'urnes cinéraire en verre qui indiquent généralement les sépultures les plus riches.

\section{CORREZZE}

\section{Afrieux, Le Leyrit (fig. 5, $\mathrm{n}^{\circ} 1$ )}

Armature de fourreau d'un coutelas miniaturisé mis au jour en 1959 dans une sépulture constituée d'un coffre renfermant une urne en verre de forme Morin-Jean 3. Le mobilier, abondant, comprenait, entre autres, une monnaie d'Antonin-le-Pieux.

La lame, disparue, devait mesurer $0,10 \mathrm{~m}$ environ. Le fourreau est en bronze avec œillets de suspension au niveau de la chappe. Il est terminé par une boulerolle en forme de bouton à godrons. La disparition des plaques permet de voir deux ajours ménagés de part et d'autre de l'entretoise.

VAZEILLES, 1961, p. 110-111 et 1962, p. 217220 - Fournier, 1963, p. 489 - Lintz, 1981 b, p. 90

\section{Argentat, Croissy (fig. $3, \mathrm{n}^{\circ} 1$ )}

Coutelas découvert en 1827 dans une sépulture constituée d'un coffre de briques renfermant deux vases en poterie. Le premier contenait les cendres du défunt et le second le mobilier. Il a aujourd'hui disparu mais E. Bombal en a publié un bon dessin.
Il est en fer forgé d'une pièce. Le dos, légèrement convexe, est prolongé d'une soie courte dont l'extrémité semble recouverte d'une bague en feuille de bronze. Le tranchant est ondulé. Le talon semble émoussé. Du fourreau ne subsiste que la bouterolle en bronze. Le dessin ne permet guère de savoir si l'élément de bronze situé à l'extrémité proximale de la lame est une chappe ou une garde. Longueur de la lame : $0,21 \mathrm{~m}$.

Lalande, 1881, p. 142-145 - Bombal, 1897 , p. 78 - MartignoN, 1941 , p. 409-414 - Lintz, 1981 b, p. 72 et 1982, p. 32

\section{Eyrein, La Jugie (fig. $3, \mathrm{n}^{0}$ 2)}

Coutelas mis au jour dans un tertre de pierres fouillé en 1936. Il appartenait à l'abondant mobilier d'une sépulture composée d'une urne en terre cuite enfermée dans un coffre de pierre. La céramique permet de dater cette sépulture de la seconde moitié du II ${ }^{e}$ s. Présenté à l'exposition "Richesses archéologiques de la Corrèze" à Uzerche.

Il est en fer, forgé d'une pièce. La soie, située dans le prolongement du dos de la lame, est brisée. Le dos est légérement concave; la lame légèrement ondulée avec le talon bien marqué mesure $0,23 \mathrm{~m}$. Sur le même plan, un cran souligne la limite de la soie et de la lame. Le même dispositif se remarque sur certains coutelas. En servant de butée, il devait permettre la fixation d'une garde rajoutée comme dans le cas du coutelas de Méasnes $\left(\mathrm{n}^{\circ} 19\right)$ ou de celui de Saint-Laurent-les-Églises $\left(\mathrm{n}^{\circ} 39\right)$.

Shadwell, 1936, p. 115-125 - Antignac, 1974 , p. $79-83$.

\section{Lestards, Coissac}

La découverte de deux coffres funéraires, exhumés vers 1880 , a livré un couteau insufisamment décrit. D'ailleurs, le Cdt Martignon l'a considéré comme douteux dans son inventaire. Disparu.

Lalande, 1882 , p. 355-362 - MarTignon, 1941, p. 409.

\section{Meymac, Le Jassonneix}

Bien que cette découverte soit signalée par le $\mathrm{C}^{\mathrm{dt}}$ Martignon, seuls un fragment d'épée et des fers de lance sont mentionnés dans une petite nécropole détruite vers 1880 . Mobilier disparu.

LaLANDE, 1881, p. 133-134 - MarTignon, 1941, p. 409. 


\section{Sterandon, Bellegarde}

Un poignard est mentionné dans une sépulture constituée par une urne en terre cuite enfermée dans un coffre funéraire.

En outre, le mobilier comprenait une hache et une clé.

Borde, 1930.

\section{SaINT-Julien-Aux-Bois, La Garde}

Une importante nécropole comprenant essentiellement des urnes en terre cuite a livré un poignard.

Archives Départementales de la Corrèze, dossier $171 \mathrm{~T}$ 1, lettre du 5-9-1846 et du 18-9-1846 - LinTz, 1981 b, p. 71.

\section{CREUSE}

8 Ahun, Ermitage Sainte-Catherine (fig. $4, \mathrm{n}^{\circ} 1$ et fig. $\left.6, \mathrm{n}^{\circ} 2\right)$

Fourreau de petit poignard découvert en 1872 , entre Ahun et le Moutier, près d'un mur en petit appareil, parmi du mobilier pouvant provenir d'un dépotoir (os d'animaux, charbons, tessons, fibule et as de Nimes). Cet objet est conservé au Musée municipal de Guéret.

Ce fourreau est en bronze, de forme triangulaire avec amorce d'œillets de suspension au niveau de la chappe. L'extrémité distale est brisée mais, au moment de sa découverte, il se terminait par un gland et mesurait $0,084 \mathrm{~m}$. L'une de ses faces possède un bouton et une bélière.

Cessac, 1873 c, p. 231-234; $1873 \mathrm{~d}$ et Dict. ms. - Janicaud, 1942, p. 264-265.

\section{ARRÈnes, Les Giraudes}

Le mobilier découvert en 1870 dans une sépulture constituée d'un coffre de pierre à double cavité comprenait divers objets bien décrits et même dessinés (cuillère, petite hache, petite pioche et petite serpe). Il est seulement fait allusion au glaive de Boismandé (Saint-Sulpice-les-Feuilles, HauteVienne), associé à une serpe. Cité à tort par le $\mathrm{C}^{\mathrm{dt}}$ Martignon.

Cessac, 1882, p. 17-22 el Dict. ms., rubrique Arrènes - Martignon, 1941, p. 410.
10 AzÉrables, Rossignol (fig. 3, $\mathrm{n}^{\circ}$ 3)

Coutelas découvert en 1850 dans une anfractuosité de rocher entre les villages de Rossignol et de la Fayolle, dans un champ dit de La Juslice. Il se trouvait près d'une urne remplie d'ossements. Conservé au Musée municipal de Guéret.

Il est forgé d'une seule pièce. Le dos légèrement convexe se prolonge par une soie brisée. La lame, ondulée et au talon bien marqué, mesure $0,27 \mathrm{~m}$. Il possède une garde en bronze. Deux éléments du fourreau subsistent; il s'agit de la bouterolle et de la chappe en feuille de bronze. Le fourreau lui-même devait être en matière périssable.

Fillioux, 1854, p. 44 et 1866, p. $185-$ Caumont, 1865 , p. 475 - Cessac, 1872; 1873 a, p. $107-109 ; 1873 \mathrm{~b} ; 1873 \mathrm{c} ; 1873 \mathrm{~d}$ et Dict. ms., rubrique Azérables - Martignon, 1941 , p. $413-415$ - LinTz, 1981 a, p. 38 et 1982, p. 31-36.

\section{Chambonchard, Prat}

La découverte, vers 1895 , d'un coffre funéraire haut de $0,80 \mathrm{~m}$, parfaitement travaillé, aurait livré des objets en or et des poignards ciselés. Le tout aurait été vendu à un bijoutier.

LAVILle, 1966.

\section{GLÉNIC, Vaumoins}

Une sépulture découverte le 24 août 1842, au lieu-dit Pras-Marly, comprenait un coffre funéraire en pierre renfermant une urne en verre à une anse. Le mobilier funéraire, datable du $\mathrm{II}^{\mathrm{e}} \mathrm{s}$. était abondant. Parmi les objets métalliques, Bonnafoux mentionne : «Deux petits fragments, plus oxydés que les autres, représentent des lames de sabre ou de couteau ". P. de Cessac a décrit ces restes. Un des fragments possédait une soie dans le prolongement du dos de la lame qui mesurait au moins $0,18 \mathrm{~m}$.

Bonnafoux, 1843 b, p. 123-127 - Cessac, 1882 , p. 268 - Martignon, 1941, p. 410.

13 Grand-Bourg (Le), Longeville (fig. $5, \mathrm{n}^{\circ} 2$ et fig. $\left.6, \mathrm{n}^{\prime \prime} 6\right)$

Coutelas miniaturisé mis au jour en 1834, au lieu-dit Bois $d u$ Lac, sous le coffre d'une sépulture à incinération de section carrée renfermant une urne en verre de forme Morin-Jean 14. Le mobilier funéraire comprenait de nombreux tessons de poteries dunt cerlaines, en sigillée, dateraient du $\mathrm{II}^{\mathrm{e}} \mathrm{s}$. Il est conservé au Musée municipal de Guéret. 
Il s'agit d'un coutelas miniaturisé à poignée de bronze et à lame de fer visible à travers les ajours d'une plaque du fourreau. La poignée de bronze, longue de $0,062 \mathrm{~m}$, est dans l'axe du dos de la lame. Le pommeau représente une tête de félin. Des godrons ornent la base de la garde et marquent la limite entre la fusée et le pommeau. Les côtés de la fusée comportent des cercles concentriques estampés.

La radiographie de cet objet montre que la poignée est obtenue par un seul jet de fonte. Le pommeau n'est pas rapporté. Aucun rivet n'est visible au niveau de la garde. La fixation poignéelame est assurée par coulée directe de la poignée de bronze sur la soie et le talon de la lame en fer.

La lame de fer, longue de $0,10 \mathrm{~m}$, possède une pointe mousse et un tranchant très légèrement ondulé, elle se prolonge par une soie courte, située dans l'axe du dos subrectiligne de la lame. L'ensemble, forgé d'une pièce, ménage un talon moins prononcé que celui de Flavignac ( $\left.\mathrm{n}^{0} 35\right)$.

Le fourreau en bronze mesure $0,118 \mathrm{~m}$. Il comporte d'un côté une plaque de bronze ornée d'un motif de rinceaux incisés. De l'autre côté, deux ajours permettent de voir la lame; il n'est pas possible de savoir si une plaque en matière périssable la protégeait à cet endroit. Une bouterolle en forme de bouton garnit l'extrémité distale du fourreau. Un crochet de suspension et l'amorce d'un second sont situés à l'entrée du fourreau. L'amorce d'un œillet de suspension prolonge l'entretoise. L'examen de la radiographie du fourreau et de ses accessoires (bouterolle, crochets, anneau de suspension) montre qu'il a été coulé d'une seule pièce.

JANICAUD, $1934 ; 1935$, p. 53-54 et 1942, p. $260-261$.

14 Janaillat, La Vacheresse 'fig. 5, no 3 et fig. 6 $\mathrm{n}^{\circ} 5$ )

Coutelas miniaturisé découvert en 1854 dans une sépulture en coffre avec urne en verre et une monnaie d'Hadrien. Conservé au Musée de Guéret (Inv. 342).

Seule la partie proximale de la lame est conservée et demeure visible sur l'un des côtés du fourreau. La garde et les quillons de la poignée en bronze sont godronnés. Il en est de même pour la fusée cassée au-dessous du pommeau. Celui-ci a disparu.

La radiographie de cet objet montre que la poignée est obtenue par un seul jet de fonte. Aucun rivet n'est visible au niveau de la garde. La fixation poignée-lame est assurée par coulée directe de la poignée de bronze sur la soie de la lame de fer.

Le moignon de la lame ne permet guère d'en indiquer les caractéristiques. La radiographie du fourreau a permis de visualiser l'espace qu'empruntait cette lame qui pouvait avoir une quinzaine de centimètres de long. La soie est située dans le prolongement de l'axe du dos.

Le fourreau de bronze, prolongé de sa bouterolle est constitué, d'un côté, d'une plaque de bronze sans décors; l'autre côté est muni d'un évidement triangulaire. De chaque côté du fourreau et à l'intérieur de celui-ci, le bronzier a ménagé une feuillure, permettant d'une part de guider et de maintenir la lame contre la plaque de bronze et d'autre part de servir vraisemblablement de support à une plaque de matière périssable destinée à protéger la lame. Anneaux et amorces d'anneaux de suspension sont visibles à l'embouchure du fourreau et dans sa partie médiane. L'examen de la radiographie semble indiquer que le fourreau a été coulé d'une seule pièce. Il mesure $0,172 \mathrm{~m}$.

Fillioux, 1854, p. 44 - Bonnafoux, 1866, p. $175-177$ - CESSAC, 1873 c, p. $224-$ MARTIGNON, 1941, p. 410 et p. 416 JANICAUD, 1942, p. 259-260.

\section{JaRNAGES}

Le Commandant Martignon signale un glaive douteux à Jarnages. A notre connaissance, seule une épée y est mentionnée. En outre, une confusion a dû se produire avec Parsac $\left(n^{\circ} 20\right)$.

Fillioux, 1869, p. $96-$ Martignon, 1941 , p. 410.

\section{Lépinas, La Terrade (fig. $3, n^{\circ} 4$ )}

Coutelas découvert en 1923, au lieu-dit Subeaufour, à $220 \mathrm{~m}$ à l'e. du hameau de La Terrade. Il était placé verticalement dans une sépulture à incinération composée d'un coffre cylindrique en granite dans lequel les cendres étaient déposées directement dans le réceptacle du socle. Le mobilier, relativement abondant, comprenait, entre autres, quatre pointes de javelot et une monnaie d'Hadrien. Il est déposé au Musée de Guéret.

Le $\mathrm{C}^{\mathrm{dt}}$ Martignon cite la commune de Lépinas à deux reprises. La deuxième mention doit faire double emploi avec la trouvaille de La Terrade. 
Ce coutelas forgé d'une seule pièce, possède une lame longue de $0,26 \mathrm{~m}$ avec un tranchant ondulé et un dos convexe. La soie, située dans le prolongement du dos, mesure $0,09 \mathrm{~m}$. Elle se termine par un bouton devant servir d'arrêt à une poignée en matière périssable.

BORdIER, 1924, p. LXXXXIII - JANICAUD, 1929 , p. 358-359-MARTignON, 1941, p. 410.

\section{Maziêre-AuX-Bons-Hommes (LA)}

Lame de poignard découverte en 1777 (?).

$$
\text { LECLER, 1902, p. } 423 .
$$

\section{MEASnes, Lavaud}

Un sabre, mis au jour dans une sépulture inédite, n'est pas décrit. Arme douteuse d'après le $\mathrm{C}^{\mathrm{dt}}$ Martignon.

Cessac, 1873 c, p. 224, n. $2 ; 1873 \mathrm{~d}-$ Martignon, 1941, p. 410.

\section{Measnes, Lavaud (fig. 3, $\mathrm{n}^{\circ} 5$ )}

Coutelas mis au jour dans une sépulture à incinération composée d'un coffre cinéraire enfermant une urne en verre (Morin-Jean 5). Le mobilier comprenait un vase en verre blanc (Morin-Jean 21), une hypossandale, une fibule émaillée et deux monnaies d'Auguste (?). Collection particulière.

Il est en fer forgé d'une seule pièce; le dos ondulé, prolongé par la soie de $0,09 \mathrm{~m}$ de long, se termine par une virole en bronze servant de pommeau. Elle porte un décor au repoussé figurant une rosace. Un cran marque la limite proximale de la lame et sert de butée à une garde en bronze. La lame ondulée, longue de $0,207 \mathrm{~m}$ a un talon bien marqué sur lequel repose la garde. Il reste, sur la lame, les vestiges d'un fourreau en fer très oxydé.

ROGER, 1929, p. 36-44

\section{Parsac}

Sur le territoire de cette commune, le $\mathrm{C}^{\mathrm{dt}}$ Martignon cite trois découvertes qu'il considère comme douteuses. Nous avons retrouvé la mention d'un sabre romain mis au jour quelques années avant 1810. L'intitulé de la note, "restes romains à Jarnages", a pu entraîner une confusion avec cette commune.

Cessac, 1861, p. 328 - Martignon, 1941, p. 410 .
21 Saint-Christophe, Le Mas-Foreau (fig. $3, n^{\circ} 7$ )

Coutelas découvert en 1848 dans une sépulture à incinération constituée d'un coffre cinéraire renfermant une urne en verre à deux anses. Le mobilier comprenait une serpe, une bague en or et une autre en fer.

Cette arme, conservée au Musée de Guéret est actuellement mutilée. C'est un coutelas dont la lame mesurait $0,24 \mathrm{~m}$ et la soie $0,13 \mathrm{~m}$. Sa forme générale est celle du coutelas de Savennes $\left(\mathrm{n}^{\circ} 31\right)$.

Fillioux, 1854, p. 44 - Cessac, $1873 \mathrm{~b}$, p. $265 ; 1872$, p. 151 ; Dict. $\mathrm{ms}$, rubrique Savennes - LiNTZ, 1982, p. 33.

\section{SAINT-Dizier-LA-Tour}

Aucune mention de glaive gallo-romain n'est connue dans la bibliographie et en particulier dans la relation que $P$. de Cessac a faite de la fouille qu'il a effectuée dans une motte féodale à la Tour-SainteAustrille. Toutefois, trois coutelas sont mêlés au matériel métallique provenant de ces fouilles. Celui de Reillac, commune de Savennes, se reconnait facilement en raison de la rondelle de cuivre rivetée sur l'extrémité distale de la soie. Il en est de même pour celui du Mas-Foreau, commune de SaintChristophe, grâce aux dimensions données par de Cessac. Le troisième est trop abîmé pour être identifié. Peut-être est-ce celui de Glénic dont on sait qu'il était au musée. La mention, par le $C^{\text {dt }}$ Martignon, de coutelas à la Tour-Sainte-Austrille est par conséquent erronnée.

Cessac, 1867 - Martignon, 1941, p. 410 - LinTz, 1982, p. 35.

\section{Saint-Dizier-Leyrenne, Le Pommier}

Une sépulture à incinération, exhumée vers 1840 comprenait une urne en verre pleine d'ossements placée dans un coffre funéraire en pierre. Elle aurait livré un poignard miniaturisé dont la gaine et le manche, longs de $0,165 \mathrm{~m}$ étaient en "cuivre ciselé». Cet objet n'est pas connu. Il aurait été vendu à Limoges. Il est fort possible que cet objet fasse double emploi avec celui que Fillioux, suivi par le $\mathrm{C}^{\mathrm{dt}}$ Martignon, signale au village du Poirier.

En effet, aucune sépulture connue, située près d'un village de ce nom, aux environs de Bourganeuf, ne correspond à cette trouvaille.

Bonnafoux, $1843 \mathrm{a}$, p. $122-123$ et $1843 \mathrm{~b}$, p. 116-117 - Fillioux, 1854, p. 44 Martignon, 1941, p. 410 et p. 416 JANICAUD, 1942, p. 264. 


\section{Saint-Dizier-Leyrenne, Font-Léon}

Une sépulture à incinération comprenait une urne en terre dans un coffre cinéraire. Près de l'urne, se trouvaient une hache et un poignard très grossier.

JANICAUD, 1954, p. III et XIX.

\section{Saint-Hilaire-Le-Château, le Bourg}

(fig. $4, \mathrm{n}^{\circ} 2$ et fig. $6, \mathrm{n}^{\circ} 4$ )

Découverte, au $\mathrm{XIX}^{\mathrm{c}} \mathrm{s}$., d'un poignard dans une sépulture à incinération. L'urne cinéraire en verre se trouvait en pleine terre, sans protection apparente. Cet objet est conservé au Musée de Guéret (Inv. 121). Le $\mathrm{C}^{\mathrm{dt}}$ Martignon place cette découverte sur la commune de Saint-Éloy.

Ce poignard miniaturisé à poignée, fourreau de bronze et lame de fer mesure $0,168 \mathrm{~m}$ de long dont $0,048 \mathrm{~m}$ pour la poignée et $0,12 \mathrm{~m}$ pour le fourreau.

La lame n'est ni oxydée ni patinée. Son aspect moderne fait penser à une restauration récente. Cette hypothèse se trouve encore renforcée par sa longueur $(0,075 \mathrm{~m})$, ce qui place sa pointe vers le milieu de l'évidement du fourreau.

La poignée possède une garde assymétrique en croix, concave vers la fusée. Celle-ci et la partie interne des quillons sont chanfreinées. La fusée se trouve dans l'axe médian de la lame; elle se termine par une boule surmontée de trois boutons formant le pommeau.

L'examen de la radiographie de cet objet montre que la poignée a été coulée d'une seule pièce puis ajustée sur la soie de la lame. Aucun rivet n'assure la fixation de la poignée sur la lame.

Le fourreau en bronze possède un crochet de suspension au niveau de la chappe. Il se termine par une bouterolle en forme de gland. Les deux plaques du fourreau, identiques, sont soulignées par un trait incisé qui ménage un espace décoré d'un rinceau.

Martignon, 1941, p. 410 et p. $417-$ JANICAUD, 1942, p. 263-264.

\section{SaINT-Hilaire-Le-ChâteaU, Laugères}

Découverte, en 1835, d'une sépulture à incinération comprenant une urne en terre cuite placée dans un coffre funéraire. Le mobilier se composait d'une pelle, d'une cuillère, de deux crampons et d'un clou long de 0,20 m. Cet objet, aujourd'hui disparu, était très abîmé à tel point que de Cessac disait à son sujet: "Je ne peux dire qu'il ressemble à celui de Reillac. Tout ce qu'on peut dire c'est qu'il était de même longueur et n'avait qu'un seul tranchant".
Cessac, 1872 , p. $151-1873$ c, p. 268 et Dict. ms., art. Savennes - Martignon, 1941, p. 410 - Lintz, 1982, p. 35.

27 Saint-Maixant, Chaussidoux (fig. $4, \mathrm{n}^{\circ} 3$ et fig. $6, \mathrm{n}^{\circ} 3$ )

Une nécropole à incinération d'une dizaine de tombes en coffres cinéraires, dont certaines avec des urnes en verre, a livré, aux environ de 1875, le fourreau d'un petit poignard. Cet objet est conservé au Musée de Guéret.

Long de 0,084 $\mathrm{m}$, il est en bronze avec anneaux de suspension au niveau de son embouchure et une bouterolle godronnée. L'un des côtés est constitué par une plaque de bronze non décorée. L'autre côté possède un évidement triangulaire, sans doutc destiné à recevoir une pièce en matière périssable. Des cercles concentriques estampés décorent la chappe $\mathrm{du}$ fourreau et des traits horizontaux annoncent l'amorce de la bouterolle.

La radiographie du fourreau montre que ce poignard avait une lame triangulaire d'environ $0,07 \mathrm{~m}$ de long et que la bouterolle est évidée. La radiographie ne révèle aucune soudure et aucune trace d'emboîtage. Ce fourreau a été obtenu par coulée d'un seul jet.

Cessac, Dict. ms., art. Saint-Maixant JANICAAU, 1942, p. 264.

\section{Saint-Martin-Sainte-Catherine, La Texonnière (fig. $4, \mathrm{n}^{\circ} 4$ )}

Coutelas miniaturisé placé parmi les cendres et ossements d'une sépulture à incinération avec coffre funéraire, mise au jour en 1907 (ou 1927?) au lieu-dit Châtreix. Le mobilier funéraire comprenait en outre une bague en argent. Cet objet n'a pas été retrouvé. Le dessin reprend celui de Janicaud.

La poignée de bronze possède une garde cruciforme aux quillons bien marqués. Le pommeau, sans doute rapporté, représente une main ouverte tenant une boule entre le pouce et l'index. La fusée est situé dans l'axe médian de l'embouchure du fourreau et non sur le côté et dans le prolongement du dos de la lame comme pour les exemplaires de Grand-Bourg ( $\left.n^{0} 13\right)$, Janaillat ( $\left.n^{\circ} 14\right)$, Flavignac $\left(n^{\circ} 35\right)$, etc.

Aucune précision ne peut être donnée sur la lame en fer si ce n'est qu'elle devait être ondulée de façon à épouser la forme du fourreau.

Ce dernier, en bronze, se termine par le départ d'une bouterolle brisée; il mesure $0,10 \mathrm{~m}$ et possède 
des cillets et anneaux de suspension situés de part et d'autre de la chappe et de l'entretoise. L'une des plaques en feuille de bronze est, semble-t-il, munie d'une agrafe; l'autre plaque est ajourée. Il n'est pas possible de savoir si les deux plaques de bronze sont reliées entre elles par des frettes ou agrafes ou si le fourreau a été coulé d'une seule pièce. Longueur totale $0,17 \mathrm{~m}$.

JANICAUD, 1930 , p. 647 et 1942 , p. 261263 - Montaigut, 1967.

\section{Saint-Martin-Sainte-Catherine, Le Theil}

(fig. $3, n^{\circ} 8$ et fig. $6, n^{\circ} 1$ )

Coutelas mis au jour, en 1976, dans une sépulture à incinération, au lieu-dit du Boisseau. Le coffre funéraire renfermait une urne cinéraire ovoïde à deux anses : elle est ornée de médaillons d'applique permettant de situer sa fabrication dans les ateliers du centre de la Gaule au cours de la première moitié du II $^{e}$ siècle. Le mobilier funéraire comprenait en outre des objets miniaturisés en fer (hache, houe, serpe, poêle à frire). Cette arme est conservée à Bourganeuf, dans la tour Zizim.

Cet objet, long de $0,375 \mathrm{~m}$, forgé d'une seule pièce, possède un dos convexe à cran servant de butée esquissé par le forgeron entre la lame et la soie. Celle-ci, longue de $0,107 \mathrm{~m}$ et placée dans le prolongement du dos de la lame, se termine par un bouton forgé qui retient un disque pouvant faire office de pommeau. La lame ondulée, au talon bien marqué, mesure $0,237 \mathrm{~m}$.

Desbordes, 1977, p. 436 - Lintz, 1981 a, p. 39 et 1982, p. 33-34.

\section{Saint-Priest d'Evaux, Louroux (fig. 3, no 9 )}

Coutelas placé à plat, contre l'urne cinéraire en terre cuite d'une sépulture fouillée par D. Dussot en 1986. Il reposait à la base de la fosse, posé sur l'arène granitique et recouvert par le résidu de la crémation. Le reste du mobilier funéraire comprend un anneau, un petit couteau en fer et de la céramique dont une assiette en sigillée. Ces éléments permeltent de dater la sépulture de la seconde moitié du ${ }_{1 r^{e}} \mathrm{~s}$. comme d'ailleurs la plus grande partie des sépultures de cette nécropole. Après restauration, cet objet sera déposé au Musée de Guéret.

Cette arme, forgée d'une seule pièce, mesure $0,41 \mathrm{~m}$. C'est le plus grand exemplaire connu de ce type d'objet. La lame, longue de $0,30 \mathrm{~m}$, relativement étroite, est large de $0,06 \mathrm{~m}$ au talon et $0,05 \mathrm{~m}$ dans sa partie renflée. Le dos, légèrement convexe, est épais de $0,008 \mathrm{~m}$ dans le milieu de sa longueur et de $0,012 \mathrm{~m}$ au talon. A cet endroit, un cran marque la limite entre le dos et la poignée. La soie, longue de $0,12 \mathrm{~m}$ prolonge le dos de la lame; son extrémité semble brisée anciennement, probablement à l'endroit où se fixait la virole.

(Inédit. Nous remercions D. Dussot de nous avoir communiqué cet objet).

\section{SavenNes, Reillac (fig. $3, \mathrm{n}^{\circ} 6$ )}

Coutelas découvert en 1870 dans une importante nécropole, lieux-dits La Couture et Mas-Giraud. Le mobilier recueilli, pour l'essentiel daté du II $^{\mathrm{e}} \mathrm{s}$., a été dessiné par de Cessac. Il comprend de nombreuses céramiques, quatre estampilles sur sigillée, un abondant matériel métallique et des monnaies à l'effigie de Trajan, Lucius-Verus et Commode. Cette arme, conservée au Musée de Guéret est actuellement très mutilée. Le dessin reproduit celui de P. de Cessac.

Le dos convexe est prolongé par une soie de $0,09 \mathrm{~m}$ placée dans son prolongement. Une petite virole de bronze servant de pommeau était rivée à chaud par martelage de l'extrémité proximale de la soie. La lame ondulée mesure $0,28 \mathrm{~m}$ et posssède un talon bien marqué.

Cesssac, 1872, p. 634-639; 1873 a, p. 106$112 ; 1873$ b, p. 246-272 el Dicl. ms., rubrique Savennes; LinTz, 1982, p. 32 et 34.

\section{Souterraine (LA), Bridiers}

Une poignée de coutelas miniaturisé serait, d'après de Cessac, au Musée de Saint-Germain.

JANICAUD, 1942, p. 262.

\section{HAUTE-VIENNE}

\section{ArnaC-la-Poste (?)}

Coffre funéraire avec urne en verre MorinJean 3 découvert en 1868. Seuls deux fragments de poignée d'épée sont signalés. A écarter.

Drouault, 1905 , p. 449 et 1906 , p. $595-$ Martignon, 1941, p. 410 - Perrier, 1964 , p. 115.

\section{Châteauponsac, Les Cros (fig. 3, n" 10)}

Coutelas découvert en mai 1962 dans une sépulture constituée d'une urne cinéraire en verre Morin-Jean 3 placée dans un coffre funéraire en pierre. Le mobilier comprenait deux sesterces (Domi- 
tien et Trajan) et de la céramique commune. Conservé au Musée Notre-Terroir à Châteauponsac.

Coutelas en fer de $0,29 \mathrm{~m}$ de longueur pour une lame de $0,20 \mathrm{~m}$. L'arme, très oxydée, est forgée d'une seule pièce avec un dos légèrement convexe et un tranchant ondulé. Le talon de la lame est bien marqué; la soie courte se trouve dans le prolongement du dos de la lame où subsistent les traces d'un fourreau en fer fortement oxydè.

Baubérot, 1964, p. 297-298.

\section{Flavignac, La Ribière (fig. 5, no 4)}

Petit coutelas dans une sépulture découverte en avril 1950 et constituée d'un coffre en granite renfermant une urne en verre de forme MorinJean 3. Les deux fragments du poignard, brisé au niveau de la garde avant l'enfouissement, étaient déposés chacun sur une anse de l'urne. Le mobilier permet de dater cette sépulture de la seconde moitié du $\mathrm{II}^{\mathrm{e}} \mathrm{s}$. Conservé à la Mairie de Flavignac.

Coutelas miniaturisé à poignée de bronze et à lame de fer visible au travers des plaques évidées du fourreau de bronze.

La poignée en bronze est dans l'axe du dos de la lame. Le pommeau représente probablement une tête de lion, retouchée au burin après coulée. Des motifs godronnés ornent la fusée et la base de la garde qui comprend en outre des chevrons incisés. Les côtés de la fusée et le quillon de la garde portent des cercles concentriques estampés.

La radiographie de cet objet montre que la poignée est obtenue par coulée. Le pommeau n'est pas rapporté. Aucun rivet n'a été observé au niveau de la garde. Une bulle d'air visible dans la concavité de la soie indique que les gaz ne se sont pas échappés lors de la coulée. Seule la coulée directe de la poignée de bronze sur la soie et le talon de la lame en fer assure la fixation de la poignée à la lame.

La lame de fer de 0,09 $\mathrm{m}$ de long, au tranchant légèrement ondulé, est prolongée de sa soie située dans l'axe du dos. L'ensemble a été forgé d'une pièce en ménageant un large talon dont la forme a été épousée par le bronzier lors de la coulée de la poignée.

Les plats de la lame devaient être protégés par les plaques du fourreau en matière périssable. Seules subsistent la chappe protégeant l'embouchure du fourreau, les frettes et une entretoise en bronze. La bouterolle a disparu. L'examen à l'œil nu et la radiographie ne permettent pas de déceler de traces de soudure, de sertissage ou d'agrafage. Il semblerait que cette partie en bronze ait été obtenue d'une pièce par coulée. Des vestiges d'anneaux de suspension flanquent l'extrémité de la chappe.

Laborderie, 1950 - Perrier, 1964, p. 89.

\section{LimoGes, Manège de cavalerie (fig. 5, $\mathrm{n}^{0} 5$ )}

Petit poignard découvert en 1845 . L'original a disparu mais on en connaît un dessin.

D'après le dessin, la poignée en bronze est dans l'axe du dos de la lame. Le pommeau représente un petit animal (panthère, chien?). Deux motifs godronnés ornent la fusée. Le quillon de la garde ressemble à celui de Grand-Bourg ( $n^{0} 13$ ). Le fourreau semble constitué de deux feuilles de bronze brisées dans leur partie dislale. Deux appendices subsistent au niveau de la chappe ainsi que le pontet sur le plat du fourreau. Deux vestiges d'anneaux à bélières sont visibles sur la partie ondulée du fourreau.

Ardant, 1859 , p. 133-135 - Martignon, 1941 , p. 412 - Perrier, 1964, p. 83.

\section{LimOGES, Usine à Gaz (fig. 4, n 5)}

Ce poignard, découvert en 1924 dans la zone s. des terrains de l'Usine à Gaz proviendrait d'une sépulture. D'après le dessin publié par F. Delage.

De la poignée, seul subsiste le pommeau cruciforme en bronze. Un rinceau incisé orne le fourreau. Au niveau de l'embouchure, des crochets de suspension supportent des glands de chêne en bronze. Sa forme triangulaire laisse supposer l'existence d'une lame de même forme longue d'environ $0,16 \mathrm{~m}$. La poignée devait se trouver dans l'axe de la lame.

Delage, 1944, p. 248-250 - Perrier, 1964, p. 83.

\section{RAZÈs(?) (fig. 5, no 6)}

Petit coutelas découvert dans un lieu non précisé de la commune, avant 1859. L'original a disparu mais on en connaît un dessin.

C'est un objet miniaturisé à poignée en bronze et lame en fer. La poignée ressemble à celle de Flavignac ( $\left.n^{0} 35\right)$. Le pommeau figure une tête de lion; incisions au burin et cercles concentriques estampés ornent la garde et la fusée.

Le fourreau en bronze se prolonge par une bouterolle. La chappe godronnée est flanquée de quatre anneaux de suspension. L'une des plaques du 
fourreau est décorée. Des points juxtaposés soulignent les côtés. Une tige de lierre terminée par une feuille, tracée suivant la même technique occupe l'extrémité distale. Un personnage debout, torse et jambes nus, est obtenu par des traits incisés, le torse est représenté de face, la tête et les jambes de profil. Il tient un objet indéterminé. Au-dessus, une inscription peut se lire TRAS ou IRAC. Un cercle estampé se trouve à l'extrémité de l'inscription.

Ardant, 1859 , p. 133-135 - Martignon, 1941, p. 412-413 - Perrier, 1964, p. 104.

\section{Saint-Laurent-Les-Églises, Le Bussin (fig. 3,} $\left.\mathrm{n}^{\circ} 11\right)$

Coutelas mis au jour en 1960, au lieu-dit $L a$ Gachade, dans une sépulture constituée d'une urne en verre Morin-Jean 3 parmi un abondant mobilier placé autour du coffre : 3 lampes, environ 25 vases, une épée, une pointe de lance, une hache, une boucle d'oreille en or et des amulettes. Dessin d'après photo.

Il est en fer forgé d'une seule pièce. Le dos légèrement concave est prolongé par la soie. Le tranchant ondulé a un talon bien marqué. Une garde de bronze bute sur le talon de la lame et sur un cran ménagé, sans doute à cet effet, entre la soie et le dos de la lame. Des traces de fourreau en fer subsistent sur les plats de la lame. Longueur de la lame: $0,248 \mathrm{~m}$, de la soie : $0,098 \mathrm{~m}$.

Perrier, 1961 , p. $12-18$ et 1964 , p. 101 . Eygun, 1961, p. 418.

\section{Saint-Martin-Terressus, Réserva (fig. 3, $\mathrm{n}^{\circ} 12$ )}

Coutelas exhumé en 1967 dans une sépulture constituée d'une urne en verre Morin-Jean 3 enfermée dans un coffre en pierre. Le mobilier comprenait un abondant matériel céramique. Le coutelas, dissocié du résidu de la crémation, reposait sur une banquette aménagée sur le pourtour de la fosse. Dessin d'après photo.

En fer, forgé d'une seule pièce, il possède un dos, légèrement concave, marqué d'un cran à sa partie distale. Il est prolongé d'une soie cassée. La lame ondulée, longue de $0,214 \mathrm{~m}$, possède un talon bien marqué.

Perrier, 1969, p. 58-62.

\section{SaInt-Sornin-Leulac, Le Monteil}

Deux coffres funéraires renfermaient chacun une urne en verre Morin-Jean 5. Le mobilier, peu abondant semble-t-il, comprenait un poignard. Disparu.

Baubérot, 1967, p. 273.

\section{Saint-SulPICE-Les-Feuilles, Boismandé}

Une sépulture constituée d'un coffre avec une urne en verre, mise au jour en 1869 , aurait livré un glaive non décrit accompagné d'une serpette. Disparu.

Beaufort, 1874, p. 23 - Cessac, Dict. ms. - Martignon, 1941, p. 410 - Perrier, 1964, p. 115.

\section{SAInT-Symphorien-Sur-Couze, La Churlerie}

Trois sépultures mises au jour en 1949 comprenaient chacune une urne en verre de forme MorinJean 3 abritée dans un coffre funéraire en granite. L'une d'elle renfermait un abondant mobilier comprenant des tessons de céramique moulée du centre de la Gaule datée du milieu du II $^{\mathrm{e}} \mathrm{s}$. et le manche en bronze d'un petit couteau.

La forme de la lame n'est pas connue. La poignée longue de $0,052 \mathrm{~m}$ possède un pommeau ellipsoïde souligné par une base annulaire. La fusée, d'abord octogonale, devient rectangulaire près de la lame brisée. Deux annelets marquent le changement de forme. Il n'est pas possible de préciser si cet objet était un simple couteau, un poignard ou un coutelas miniaturisé.

\section{Perrier, 1951.}

\section{Thouron, Tournadis}

Un caveau mesurant $2,80 \mathrm{~m}$ sur $1,72 \mathrm{~m}$, découvert en 1905 près d'un coffre funéraire, contenait un fer de lance et un coutelas. L'original a disparu mais, d'après une photo publiée en 1906, il s'agit d'un coutelas forgé d'une seule pièce qui ressemble à celui du Bussin, commune de Saint-Laurent-les-Églises (no 39$)$.

La lame au dos convexe, longue de $0,22 \mathrm{~m}$, est brisée à son extrémité. La soie, longue de $0,08 \mathrm{~m}$, prolonge le dos de la lame.

Anonyme, Dolmen-Club de Bellac, 1906, p. 43-46 et 1907, p. 4 et p. 33 - LecLer, 1909 - Perrier, 1964, p. 98. 


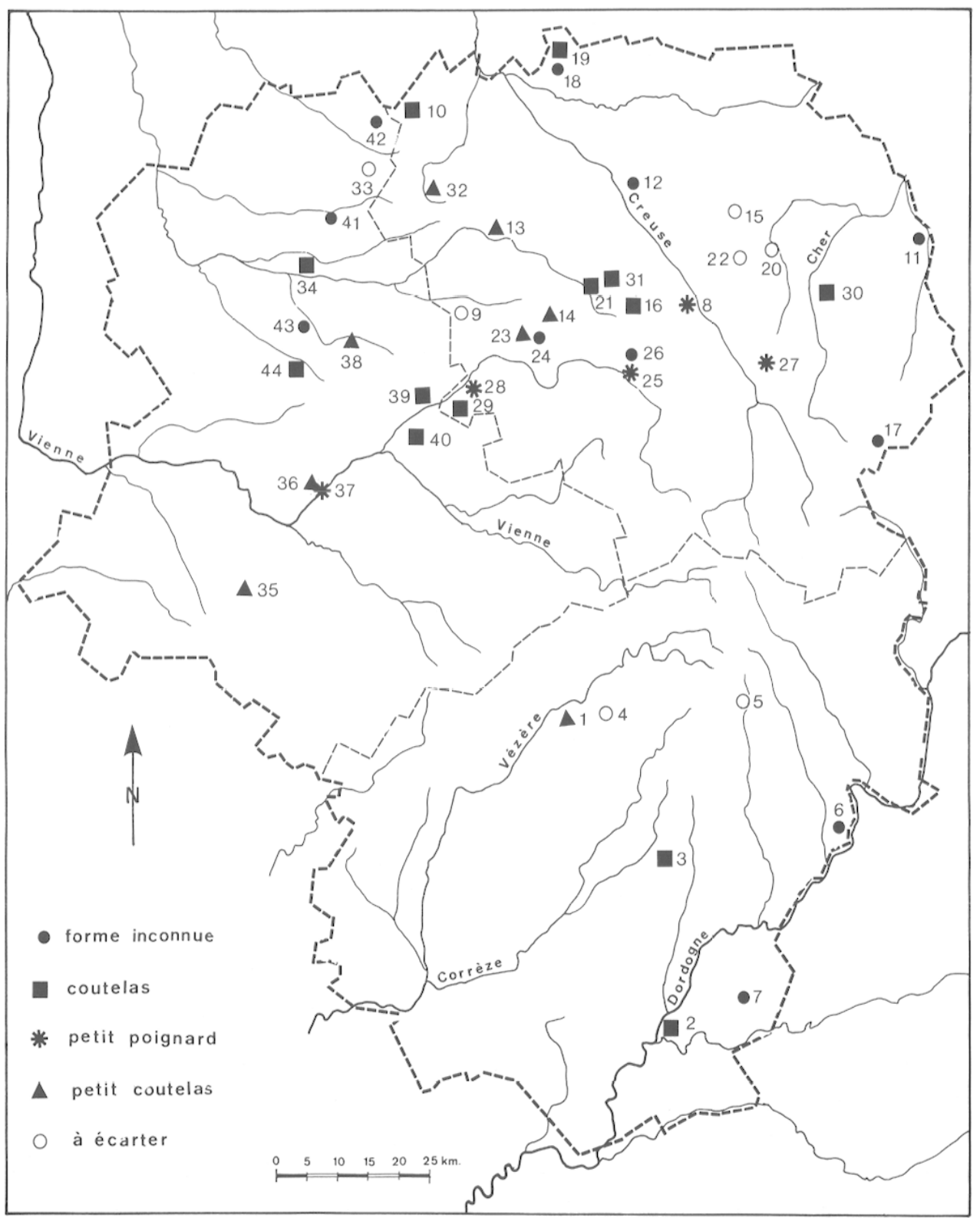

Fig. 7 - Carte de répartition des coutelas et poignards en Limousin (les numéros renvoient à l'inventaire). 


\section{BIBLIOGRAPHIE}

Amy (R.), Duval. (P.-M.), Formigŕ (J.), Picard (Ch. et G.-Ch.) et Piganiol (A.), 1962 - L'arc d'Orange, XVe suppl. à Gallia, èd. C.N.R.S.

Antignac (J.-L.), 1974 - Les sépultures gallo-romaines de la Jugie, 19, Eyrein, Lemouzi, no 49, p. 79-83.

Ardant (M.), 1859 - Communication, Bull. de la Soc. Arch. el Hist. du Limousin, IX, p. 133-135.

Armand-Caillat (L.), 1957 - Note sur un type de clef galloromaine en bronze dont le manche figure une main, Rev. Arch. de l'Est, 8, 3-4, p. 326-329.

Aymard (J.), 1951 - Les chasses romaines des origines à la fin du siècle des Antonins, Paris, 614 p.

BAubŕrot (R.), 1950 - Les découvertes de la Bussière-Étable à l'Institut de France, Bull. de la Soc. Arch. el Hist. du Limousin, 63, p. 130-146.

- 1964 - Communication, Bull. de la Soc. Arch. et Hisl. du Limousin, XCI, p. 273 et p. 297-298.

- 1967 - Deux coffres funéraires gallo-romains au Monteil, commune de Saint-Sornin-Leulac, Bull. de la Soc. Arch. el Hist. du Limousin, XCIV, p. 273.

Braufort (E. de), 1874 - Congrès Arch. de France, Châteauroux, 1873, p. 23.

BÉrarn (G.), 1961 - La nécropole gallo-romaine de la Calade à Cabasse (Var), Gallia, 19, 1, p. 104-158.

Bombal (E.), 1897 - Recherches sur la villa gallo-romaine de Longour, Bull. de la Soc. des Lettres, Sc. et Arts de la Corrèze, XIX, p. 58-103.

Bonnafoux (J.-F.), 1843a - Description de quelques tombeaux romains découverts dans le département de la Creuse, Mém. de la Soc. des Sc. Nat. el Arch. de la Creuse,

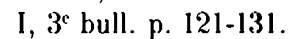

- 1843 b - Archéologie, Mém. de la Soc. des Sc. Nal. el Arch. de la Creuse, I, 3e bull., p. 107-117.

- 1866 - Sépultures gallo-romaines de Janaillac, Congrès Arch. el assises scient. de Guérel, p. 175-177.

BordF: (J.), 1930 - Une découverte archéologique, Bull. de la Soc. des Lettres Sciences et Arts de la Corrèze, 47, p. 170174 et $220-225$.

Bordier (D.), 1924 - Communication, Mém. de la Soc. des Sc. Nal. el Arch. de la Creuse, XXII, p. I.xxxxin.

Brett-Mal.her (D.), 1971 - La civilisation de la Tène I en Champagne. Le faciès marnien, $23^{r}$ Suppl. à Gallia, éd. C.N.R.S.

Breuil, (H.), 1917 - Représentations d'armes ibériques sur les monuments romains de Provence, Revue Archéologique, II, p. 68 et suiv.

Caumont (A. de), 1865 - Communication, Congrès Arch. de France, XXXII" session, Gueret, p. 475.

Cessac (P. de), 1861 - Restes romains de Jarnages, Mém. de la Soc. des Sc. Nat. et Arch. de la Creuse, III, $3^{\text {r bull., }}$ p. 328 .
- 1867 - Rapport sur les fouilles exécutées dans les buttes de la Tour-Saint-Austrille pendant l'automne de 1865, Mémoires lus à la Sorbonne dans les séances extraordinaires du Comité Impérial des Travaux IIstoriques et des Sociëtés Savantes - Archéologie, p. 215-234.

- 1872 - Le cimetière gallo-romain de Reillac, près Guéret (Creuse), Bull. monumental, $38^{\mathrm{e}}$ vol., p. 634-659.

- 1873 a - Communication, Bull. de la Soc. Nat. des Ant. de France, IV, p. 106-112.

- $1873 \mathrm{~b}$ - Le cimetière gallo-romain de Reillac, commune de Savennes, canton de Guéret (Creuse), Mém. de la Soc. des Sc. Nal. el Arch. de la Creuse, IV, 2, p. 246-272.

- $1873 \mathrm{c}$ - Les divers modes de sépulture sur le territoire de la Creuse, Mém. de la Soc. des Sc. Nal. el Arch. de la Creuse, IV, 2, p. 215-245.

- $1873 \mathrm{~d}$ - Les divers modes de sépultures sur le territoire de la Creuse, Bull. monumental, $39^{\mathrm{e}}$ vol., p. 127-161.

- 1882 - Une sépulture double de l'époque de la domination romaine, Mém. de la Soc. des Sc. Nat. et Arch. de la Creuse, V, 1, p. 17-22.

- s.d. - Dictionnaire archéologique de la Creuse, Manuscrit déposé aux archives départcmentales de la Creuse.

Chopetin (Cl.) et Périchon (R.), 1973 - Céramiques celtiques à décor estampé d'Aulnat, Rev. Arch. du Centre, XII, 3-4, p. 307-314.

Collectif 1981 - La grotte des Planches à Arbois (Jura), Gallia Préhistoire, 24, 1, p. 145-200.

Cousssin (P.), 1923 - Les armes figurées sur les monuments romains de la Gaule méridionale, Rev. Arch., II, p. 2987.

- 1924 - Les frises de l'arc d'Orange, Rev. Arch., p. 2954.

- 1927 - Les armes figurées sur les monuments grecs, étrusques et romains, Rev. Arch., I, p. 171-174.

Davin (P.), et. Garet (C.), 1974 - Le site gallo-romain de Muron, Roccafortis, $2^{e}$ série, 3, p. 111-112.

De Laet (S.), Van Doorselaer (A.), Spitaels (P.) et Thoen (H.), 1972 - La nécropole gallo-romaine de Blicquy, Bruges, 2 vol.

DÉchrifter (J.), 1904 - Les fouilles du Mont-Beuvray de 1897 à 1904, Paris-Autun.

- 1927 - Manuel d'archéologie...; IV, Second Age du fer ou époque de La Tène.

DFisge (F.), 1944 - Vestiges de la ville gallo-romaine à l'usine à gaz de Limoges - III. Poignard antique, Bull. de la Soc. Arch. et Hist. du Limousin, LXXX, p. 248250.

Desbordes (J.-M.), 1977 - Informations archéologiques, Gallia, 35, p. 436.

Devauges (J.-B.) el Cazauran (M.-C.), 1981 a - Les 
céramiques ocellées en Gaule de la fin de l'indépendance à l'époque gallo-romaine, Rev. Arch. de l'Est, 32, 1-2, p. 89-119.

- $1981 \mathrm{~b}$ - Informations archéologiques, Gallia, 39, p. 423.

Deyber (A.), 1986 - Contribution à l'étude de la guerre à la fin de l'époque de La Tène : l'emploi de l'armement celtique en Gaule au I $^{\text {er }}$ siècle de notre ère. Aquitania, suppl. 1, p. 338.

Drack (W.), 1972/73 - Waffen und Messer der Hallstattzeit aus dem Schweizerischen Mittelland und Jura, Annuaire de la Soc. Suisse de Préhist. el d'Arch., 57, p. 119-168.

Drouault (R.), 1905 - Monographie du canton de SaintSulpice-les-Feuilles, Bull. de la Soc. Arch. et Hist. du Limousin, LIV, p. 449 et LV, p. 410.

Dumoulin (A.), 1964 - Découverte d'une nécropole galloromaine à Apt (Vaucluse), Gallia, 32, p. 87-110.

- 1965 - Les puits et fosses de la colline Saint-Jacques à Cavaillon (Vaucluse), Gallia, 33, p. 1-85.

Espérandieu (E.), 1907 - Recueil général des bas-reliefs, stalues et busles de la Gaule romaine, I, Narbonnaise.

Espérandieu (E.), et Rolland (H.), $1959-$ Bronzes antiques de la Seine-Maritime, XIII ${ }^{\mathrm{e}}$ suppl. à Gallia, èd. C.N.R.S.

Eygun (F.), 1933 - Le cimetière gallo-romain des Dunes à Poitiers, Mém. de la Soc. des Anl. de l'Ouest, XI, 232 p.

- 1961 - Informations archéologiques, Gallia, 19, p. 418419.

- 1965 - Informations archéologiques, Gallia, 23, p. 358359 et fig. 17.

Faidfr-Ffytmans (G.), 1957 - Recueil des bronzes de Bavai (Nord), VIII suppl. à Gallia, éd. C.N.R.S.

Fellmann (R.), 1955 - Basel in römischer Zeil, Birkhaüser Verlag, Basel.

Feugère (M.), 1985 - Les fibules en Gaule méridionale, de la Conquêle à la fin du ve s., $12^{`}$ suppl. à la Rev. Arch. de Narbonnaise.

Furlioux (A.), 1854 - Lettre à A. Duchalais, Mém. de la Soc. des Sc. Nat. et Arch. de la Creuse, II, $1^{\text {er }}$ bull., p. 35-51.

- 1866 - Note...sur un glaive antique du musée de Guéret, Congrès Arch. et Assises scientifiques de Guéret, p. 185.

- 1869 - Les urnes cinéraires du département de la Creuse, Mémoires lus à la Sorbonne dans les séances extraordinaires du Comité Impérial des Trav. Hist ..., p. 3 du tiré à part.

Fournier (P.-F.), 1963 - Informations archéologiques, Gallia, 21, p. 489.

Galliou (P.), 1981 - Sépultures el coulumes funéraires en armorique romaine, Thèse de doctorat de $3^{\mathrm{e}}$ cycle, Université de Paris IV, dactylographié.

Gendron (Ch.) et Gomez de Soto (J.), 1986 - Le sanctuaire pré-romain de Faye-l'Abbesse (Deux-Sèvres), Aquitania, suppl. 1, p. 91 .

Grot (P.-R.), 1969 - Au seuil de l'histoire, IIistoire de la Bretagne, Privat, p. 62-63.

Guillaumet (J.), 1975 - Les séries protohistoriques de Champagne au musée Rollin, Mém. de la Soc. Eduenne, LIII, 1, p. 21-56.

Janicaud ( $D^{r}$ G.), 1929 - Mélanges archéologiques : Sépultu- res, Mém. de la Soc. des Sc. Nat. et Arch. de la Creuse, XXIV, 3-4, p. 358-359.

- 1930 - Mélanges archéologiques. Période gallo-romaine. VII. sépultures, Mém. de la Soc. des Sc. Nat. el Arch. de la Creuse, XXIV, 5, p. 645-647.

- 1934 - Communication, Mèm. de la Soc. des Sc. Nat. et Arch. de la Creuse, XXV, 3, p. Lxxxvi.

- 1935 - Communication, Mém. de la Soc. des Sc. Nat. et Arch. de la Creuse, XXVI, 1, p. 53-54.

- 1942 - Mélanges archéologiques, $9^{e}$ série : Poignards gallo-romains de la Creuse, Mém. de la Soc. des Sc. Nat. et Arch. de la Creuse, XXVIII, 2, p. 258-265.

- 1943 - La Creuse gallo-romaine. Les sépultures, Mém. de la Soc. des Sc. Nal. et Arch. de la Creuse, XXVIII, 3, p. 426-463.

- 1954 - Communication, Mém. de la Soc. des Sc. Nat. et Arch. de la Creuse, XXXII, 1, p. II et XIX.

Kersers (B. de), Marguerye (de) et Guère (R. de la), 1891 - Sépulture de Bourges, Mém. de la Soc. du Centre, 18, p. 51-76.

KiLLIAN (K.), 1970 - Zum beginn der Hallstattzeit in Italien und im Ostalpenraum, Jahrbuch des römisch-germanischen Zentralmuseums, Mainz, 17, p. 63-83.

Kruta (V.), 1971 - Le trésor de Duchcov dans les collections tchécoslovaques.

Labordarie (P. de), $1950-$ La sépulture gallo-romaine de Flavignac, Limoges, $30 \mathrm{p}$.

LALANDE (P.), 1881 - Sépultures gallo-romaines dans la Corrèze, Bull. de la Soc. Sc., Hist. el Arch. de la Corrèze, III, p. 127-160.

- 1882 - Note au sujet de lampes romaines, Bull. de la Soc. Sc., Hist. et Arch. de la Corrèze, IV, p. 355-362.

Laville (L.), et Texier (Abbé C.), 1966 - Un grand ossarium découvert à Prat, commune de Chambonchard, Mém. de la Soc. des Sc. Nat. et Arch. de la Creuse, 36, 1, p. 6569.

LeBer (P.), 1959 a - Catalogue des collections archéologiques de Besançon. V. Les bronzes figurés, Annales littéraires de l'Université de Besançon, vol. 26.

- 1959 b - Note additionnelle, Rev. Arch. de l'Est, X, p. 352-354.

- 1961 - Mains féminines en bronze tenant un objet arrondi, Reu. Arch. de l'Est, XII, 4, p. 278-283.

Lecler (Abbé A.), 1902 - Dictionnaire topographique, archéologique et historique de la Creuse, Limoges, $840 \mathrm{p}$.

LeCLer (Ch.-André), 1909 - Monographie de la commune de Thouron, $2^{\mathrm{e}}$ édition.

Lequément (R.), 1983 - Informations archéologiques, Gallia, 41, p. 476.

Lerat (L.), 1977 - La Gaule romaine, Coll. U2, Armand Colin.

Lintz (G.), Loulergue (M.) et Urien (P.), 1979 - Découverte d'une sépulture gallo-romaine à Aubusson, Mém. de la Soc. des Sc. Nat. et Arch. de la Creuse, 40, 2, p. 299311.

Lintz (G.), 1981 a — La tombe gallo-romaine (en Limousin), Archéologia, no 157, p. 35-39.

- $1981 \mathrm{~b}$ - Carte archéologique de la Gaule romaine, fasc. 16, département de la Corrèze, éd. C.N.R.S.

- 1982 - Le Glaive d'Azérables, Études Creusoises III Pages Sostraniennes pour saluer René Chatreix, p. 31-36. 
- 1987 - Les objets domestiques dans les sépultures gallo-romaines de la Creuse, à paraître dans Mélanges A. Carriat, Guéret.

Majurel (R.), 1964 - Manches historiés en bronze de la villa rustica des Orbies-Mirvaux, Ogam, XVI, 1-3, p. 90-94.

Martignon (L.), 1941 - Glaives à lame ondulée trouvés dans le Limousin, Bull. du comité des travaux Hisl., 19361937, p. 407-422.

Mennessier (Chr.), 1973 - Tombes gallo-romaines du Limousin : traitement graphique de l'information, Recherches d'Archéologie celtique et gallo-romaine, E.P.H.E., IVe section, p. 83-95.

Montaigut (Chanoine de), 1967 - Communication, Mém. de la Soc. des Sc. Nat. el Arch. de la Creuse, 36, 2, p. xxxIX$\mathbf{x x x x}$.

Perrier (J.), 1951 - Les sépultures gallo-romaines de la Churlerie, commune de Saint-Symphorien, Bull. de la Soc. Arch. et Hist. du Limousin, 83, p. 371-377.

- 1961 - Notes d'archéologie gallo-romaine. Sépulture du Bussin, commune de Saint-Laurent-les-Églises, Bull. de la Soc. Arch. et IIist. du Limousin, LXXXVIII, p. 1218.

- 1964 - Carte archéologique de la Gaule romaine, fasc. 14, déparlement de la Ilaute-Vienne, éd. C.N.R.S.

- 1969 - Notes d'archéologie gallo-romaine. Sépulture de Réserva, commune de Saint-Martin-Terressus, Bull. de la Soc. Arch. et Hist. du Limousin, XCVI, p. 58-62.

- 1970 - Sépultures gallo-romaines de Saint-Méard, Bull. de la Soc. Arch. et Hist. du Limousin, 97, p. 31-44.

Pianson (E.) el alii, 1982 - La néeropole gallo-romaine des Bolards, Nuits-Saint-Georges, Paris, éd. du C.N.R.S.
Reyniers (C.), 1959 - Un grand couteau à manche orné trouvé à Granges-le-Bourg (Haute-Saône), Rev. Arch. de l'Est, X, p. 348-354.

Roger (Fr.), 1929 - Boîtes funéraires ovoïdes. Le cimetière gallo-romain de Lavaud de Méasnes (Creuse), Mém. de la Soc. des Ant. du Centre, XLIII, p. 35-44.

Rou.tand (H.), 1977 - L'arc de Glanum, XXXI' suppl. à Gallia, èd. C.N.R.S.

Roux (M.), 1985 - Algorithmes de classification, Masson.

Salin (E.), 1951 - Le mobilier funéraire de la Bussière-Étable près Châteauponsac, Haute-Vienne, Monuments Piol, 45, p. 89-115.

SCHAEFFER (F.-A.), 1930 - Les tertres funéraires préhistoriques de la forêl de IIaguenau, vol. II, Les tumulus de l'Age du Fer.

Schonbergrer (H.), 1953 - Provinzialrömische Gräber mit Waffenbeigaben, Saalburg Jahrbuch, 12, p. 53-56.

Shadwri.r. (N. L.), 1936 - Un tumulus gallo-romain de la '2e moitié du II siècle, Bull. de la Soc. Sc., Hisl. el Arch. de la Corrèze, LVIII, p. 115-125.

Tisserand (G.), 1980 - Les ex-voto du site de Flavier à Mouzon, Rev. Arch. de l'Est, 31, p. 61-73.

Ulbert (G.), 1959 - Die römischen Donau-Kastelle Aislingen und Burghöfe, Berlin.

Van Doorselear (A.), 1965 - Les nécropoles d'époque romaine en Gaule Septentrionale, Dissertationes Archaeol. Gadenses, vol. X.

Vazeilles (M.), 1961 - Deux sépultures gallo-romaines du $\mathrm{II}^{\mathrm{e}}$ siècle en Corrèze Moyenne, Bull. de la Soc. des Let. Sc. et Arts de la Corrèze, LXV, p. 110-114.

- 1962 - Le Pays d'Ussel, Tulle, Imp. du Corrézien. 